\title{
Dewatering of foam-laid and water-laid structures and the formed web properties
}

\author{
Jani Lehmonen (1D) Elias Retulainen · Jouni Paltakari • Karita Kinnunen-Raudaskoski • \\ Antti Koponen
}

Received: 29 May 2019/Accepted: 6 November 2019/Published online: 16 November 2019

(C) The Author(s) 2019

\begin{abstract}
The use of aqueous foams as a carrier fluid for pulp fibers instead of water has re-emerged in the paper and board industry in recent years. In foam forming, a surfactant is needed to reduce the surface tension of the carrier liquid and to create foam as a process fluid and flowing medium. This presents the following questions: (1) How do the water forming and foam forming processes differ? (2) How do the obtained wet/dry fibre sheets differ after forming and after wet pressing? (3) Which differences in the process behavior and sheet properties are due to the surfactant, and which are due to the presence of air bubbles in the flowing medium? The answers to these questions were sought by using an experimental academic approach and by applying a special dynamic
\end{abstract}

J. Lehmonen $(\varangle) \cdot$ E. Retulainen · A. Koponen VTT Technical Research Centre of Finland Ltd, Koivurannantie 1, 40400 Jyvaskyla, Finland e-mail: jani.lehmonen@vtt.fi

E. Retulainen

e-mail: elias.retulainen@vtt.fi

A. Koponen

e-mail: antti.koponen@vtt.fi

K. Kinnunen-Raudaskoski

Paptic Ltd, Tekniikantie 2D, 02150 Espoo, Finland

e-mail: karita.kinnunen@paptic.com

J. Paltakari

Aalto University, Vuorimiehentie 1, 02150 Espoo,

Finland

e-mail: jouni.paltakari@aalto.fi vacuum assisted sheet former. Although foams are much more viscous than water, dewatering times were found to be approximately equal in water and foam forming at higher vacuum levels. The hydrodynamic resistance of sheet was approximately constant during water forming, while in foam forming resistance was initially even smaller than in water forming but it increased with time, being substantially higher at the end of the forming process. In certain cases, surfactant alone was found to have a similar, albeit often lower, effect on the sheet properties of foam. Surfactant improved sheet dryness (both after forming and wet pressing), lowered density, and lowered strength properties also in water forming. Foam, on the other hand, had a crucial effect particularly on certain structural properties such as formation and porosity. The difference between water and foam-laid sheets typically reduced in line with higher wet pressing pressure. This suggests that the role of surface tension and foam bubbles in controlling interfiber contact is overridden by wet pressing pressure. Thus applying foam as a carrier fluid has characteristic effects both on the papermaking process and the end product properties. The main features of foam forming can be explained by the chemical effects caused by the surfactant, and the structural effects caused by the foam bubbles. 


\section{Graphic abstract}
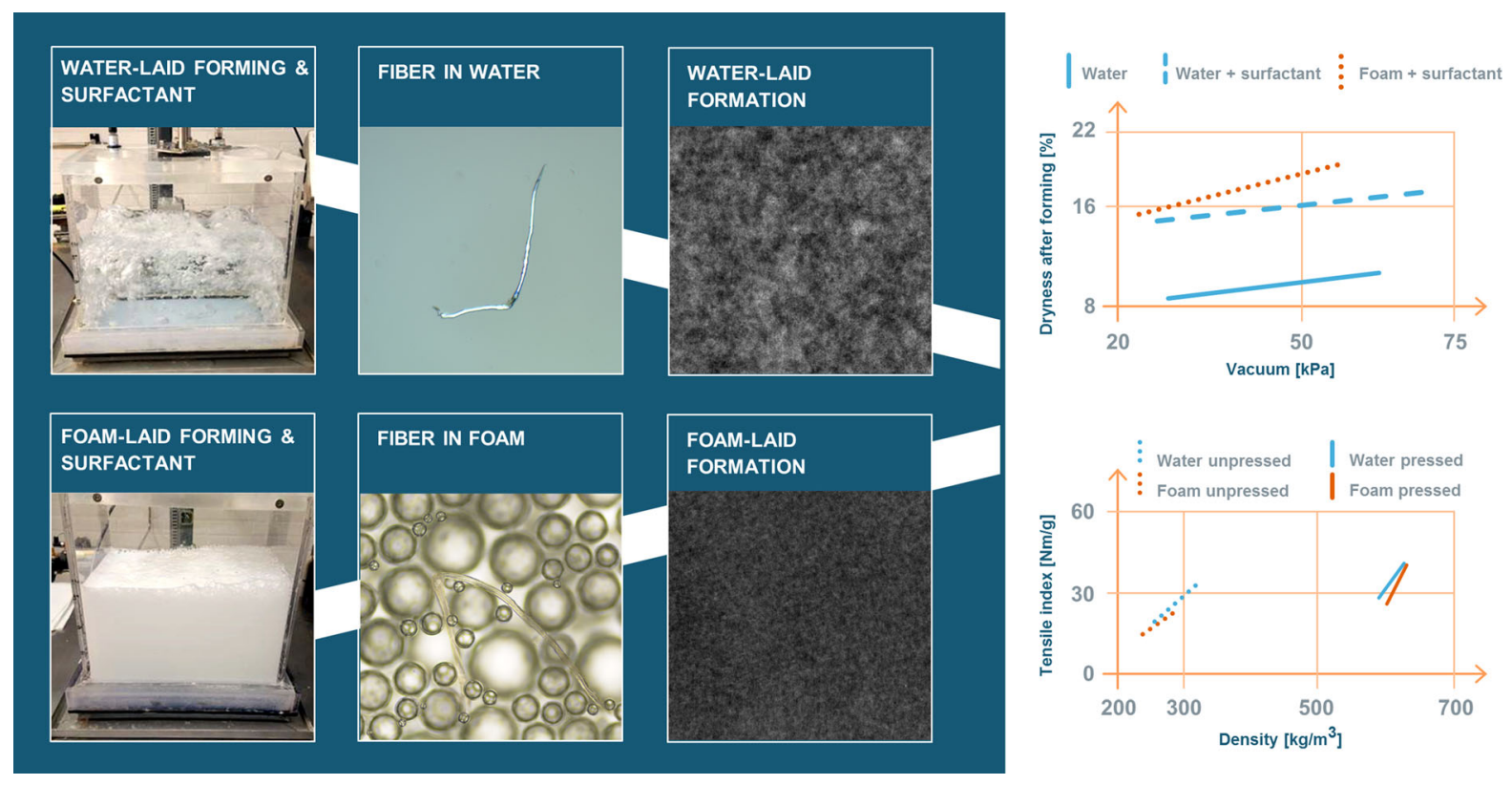

Keywords Foam forming - Water forming · Dewatering $\cdot$ Structural properties $\cdot$ In-plane strength properties $\cdot$ Interfiber contact $\cdot \mathrm{z}$-strength

\section{Introduction}

Aqueous foam is an excellent medium for transporting materials in a dispersed state. Foam is used for this purpose for example in the non-woven industry for carrying long fibers (Hanson 1977), for displacing and transporting oil in enhanced oil recovery (Farajzadeh et al. 2012), and transporting chemicals in remediation of soils (Geraud et al. 2015). Moreover, air bubbles are widely used for separation of mixtures through flotation (Lemlich 1972).

Due to jamming of the air bubbles, foams have a yield stress when the air content exceeds ca. $65 \%$ (Saint-Jaimes and Durian 1999). Foams are also much more viscous than water. Figure 1a shows a typical viscosity-air content curve at constant temperature and shear rate. Viscosity increases initially slowly with increasing air content, but above 55\% air content the increase in viscosity is very rapid. The existence of yield stress and relatively high viscosity are key properties of aqueous foams in material transport.
Foams are generally pseudoplastic power law fluids by nature. Figure $1 \mathrm{~b}$ shows as an example the shear rate dependence of a foam with an air content of $70 \%$. At low shear rates foams thus have high viscosity, but at high shear rates viscosity is considerably lower due to strong shear-thinning, leading to, for example, improved pumping properties.

The use of aqueous foams as a carrier fluid for pulp fibers instead of water was demonstrated in the paper industry in the 1970s. The forming process, known as the Radfoam process (Radvan and Gatward 1972; Punton 1975), was found to improve some product properties, particularly formation, but also bulk, in non-woven and paper applications (Kidner 1974; Smith and Punton 1975; Smith et al. 1974; Punton 1975; Riddell and Jenkins 1976). In foam forming, the foam significantly decreases the mobility of fibers due to its high viscosity, which can considerably exceed that of water. On the other hand, fibers inside the foam are locked between the bubbles, which attach to the fiber walls, thus reducing fiber entanglement (Punton 1975, see also Fig. 2). As a result, the reflocculation tendency of fibers is low, and foam forming gives excellent formation also with long wood pulp fibers (Hanson 1977; Lehmonen et al. 2013). Foam forming typically works best at an air content of approximately 60-70\% (Punton 1975), but it has recently been shown 
(a)

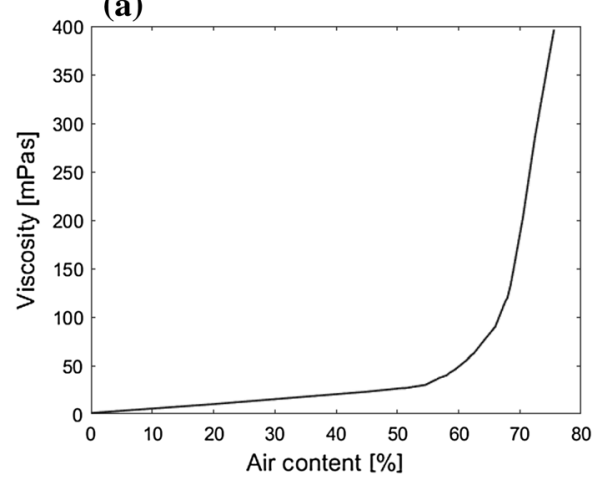

Fig. 1 a Foam viscosity (at unreported constant temperature and shear rate) as a function of air content (Punton 1975a). b Viscosity as a function of shear rate for an SDS foam (Jäsberg et al. 2015, 2017). Air content $70 \%$, temperature $25^{\circ} \mathrm{C}$. The

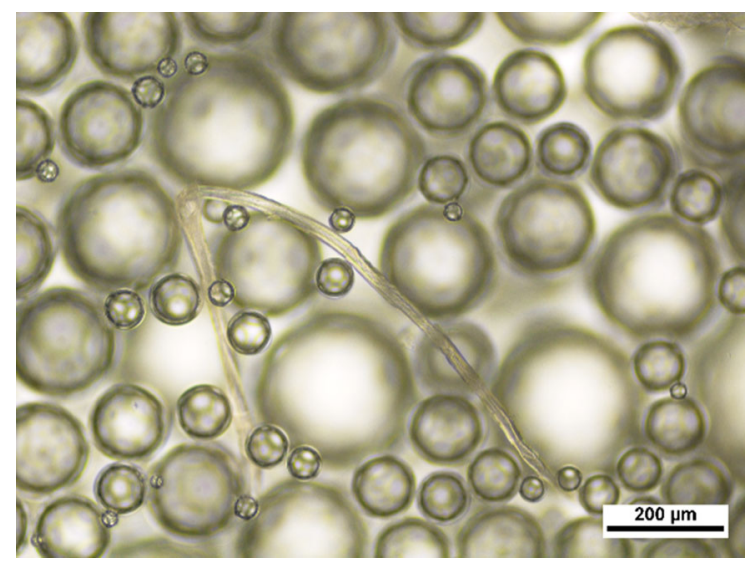

Fig. 2 A wood fiber in foam. The geometric restrictions caused by the bubbles affect the location, shape and orientation of the fiber

to also work well at as low as $30-40 \%$ air content (Torvinen et al. 2015; Koponen et al. 2018).

Foam forming offers possibilities for cost saving and environmental impact reduction through better energy and water efficiency (Smith and Punton 1975; Lehmonen et al. 2013) and higher dryness levels after the forming section (Smith et al. 1974; Wiggins 1973; Torvinen et al. 2015). Perhaps even more importantly, foam forming enables widening of the product portfolio with highly porous structures (Madani et al. 2014; Jahangiri et al. 2014; Alimadadi and Uesaka 2016; Pöhler et al. 2016; Li et al. 2017; Burke et al. 2019) and non-woven sheets composed of long fibers (Koponen et al. 2016a, b).

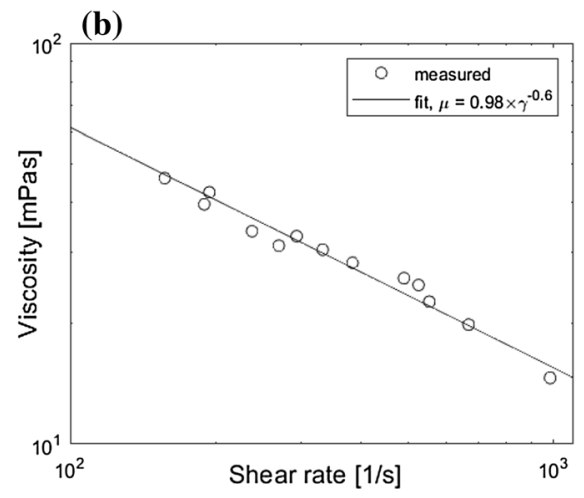

solid line shows the fit of power law $\mu=K \gamma^{n}$ to the measurement points. The foam is strongly shear-thinning, with a viscosity clearly above that of water at $1.0 \mathrm{mPa} \mathrm{s}$

There is a shortage of academic studies that would help understanding the form forming. The works on rheology of foams has concentrated on dry foams with relatively high air content, usually higher than $85 \%$. The air content in foam forming is much lower; it varies in the literature between 30 and $80 \%$, being typically 50-70\%. Moreover, the rheology of particleladen foams has been studied mainly in the presence of small particles (Thondavadl and Lemllich 1985; Cohen-Addad and Höhler 2014), and there are few studies where the size of particles has been similar or bigger than the bubble size. The rheology of fiberladen foams has been studied only in Jäsberg et al. $(2015,2017)$. While there are several studies on foam flow through various porous materials, few academic studies exist on the flow of foam through fibrous porous materials (Hirt et al. 1990). The situation is better with applied studies, and many practical aspects of foam forming have been studied especially during the last decade, also in pilot environments. The parameter space of these studies is usually rather limited for making general conclusions on foam forming. A recent review of applied studies is provided in the doctoral thesis of Kinnunen-Raudaskoski (2017).

The aim of this work is to better understand foam forming by using an experimental academic approach and by applying a special dynamic vacuum assisted sheet former. We try to give a light to some fundamental questions related to foam forming. The three questions we would like to answer are: (1) How do the water forming and foam forming processes 
differ? (2) How do the obtained wet/dry fibre sheets differ after forming and after wet pressing? (3) Which differences in the process behavior and sheet properties are due to the surfactant, and which are due to the presence of air bubbles in the flowing medium? These questions will be answered in this paper in great detail using realistic vacuum and wet pressing levels. Here the main experimental variables are surfactant dosage, forming vacuum level, and wet pressing level.

\section{Materials and methods}

Pulp and chemicals

Industrial grade gently refined and bleached chemical softwood pulp was used in the experiments. The fiber properties were characterized using a fiber analyzer (FibreMaster), and drainability was measured using the Schopper-Riegler method according to ISO standard 5267-1:1998. The length-weighted fiber length and width were $2.2 \mathrm{~mm}$ and $29 \mu \mathrm{m}$, respectively, fiber coarseness was $142 \mu \mathrm{g} / \mathrm{m}$, and drainability was $18^{\circ} \mathrm{SR}$. Sodium dodecyl sulfate (SDS), an anionic and soluble surfactant with a molecular weight of $289.4 \mathrm{~g} / \mathrm{mol}$, was used as the surface-active agent. The fairly low molecular weight of SDS leads to good foamability in foam generation (Lappalainen and Lehmonen 2012).

The surface tension and turbidity of an SDS solution made from tap water are shown as a function of SDS dosage in Fig. 3. The hardness of the tap water, measured using a Hach Lange DR3900 spectrophotometer according to the Calmagite Colorimetric

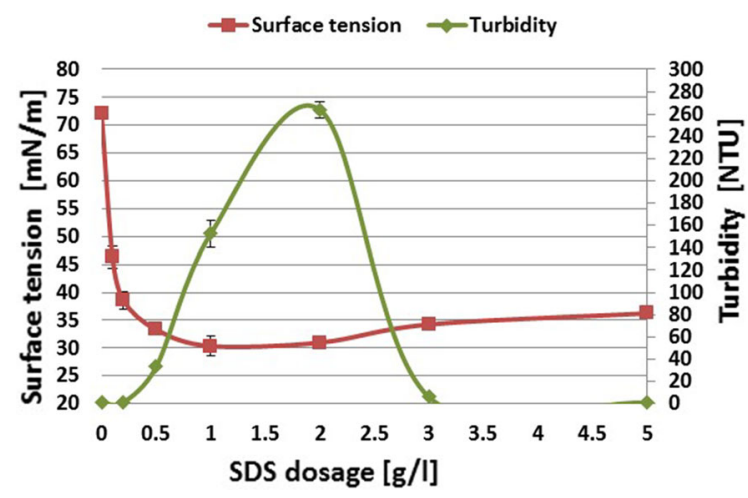

Fig. 3 Surface tension and turbidity of an SDS solution made from tap water as a function of SDS dosage at $25^{\circ} \mathrm{C}$
Method, was $3.7-4.2^{\circ} \mathrm{dH}$. Turbidity of the solution was measured with a Hach 2100AN IS Turbidimeter according to ISO standard 7027. Surface tension was measured with a KRÜSS Digital Tensiometer K9. The dosage levels of SDS were selected based on the critical micelle concentration of SDS and the surface tension and turbidity of an SDS solution were characterized before and after the critical micelle concentration. The critical micelle concentration for SDS is $2.38 \mathrm{~g} / \mathrm{l}$ in ion-exchanged water at $25^{\circ} \mathrm{C}$ and at atmospheric pressure. Due to the presence of ions, the critical micelle concentration is here considerably lower. Importantly, the turbidity indicates the level of precipitation of ions and SDS molecules in the solution. These precipitated aggregates may act as defoamers and decrease the foamability of SDS solutions (Denkov 2004; Denkov and Marinova 2006). It is also notable that the effect of SDS on viscosity is rather small. At an SDS dosage of $4 \mathrm{~g} / \mathrm{l}$, the viscosity of the SDS solution is less than $4 \%$ higher than for pure water (Kushner et al. 1952).

\section{Dynamic vacuum-assisted sheet former}

The water-laid and foam-laid papers were formed using a dynamic vacuum-assisted sheet former (Lappalainen and Lehmonen 2012; Lehmonen et al. 2017). This forming device (see Fig. 4) differs from conventional sheet formers in having faster water removal and larger sheet size $(210 \mathrm{~mm} \times 300 \mathrm{~mm})$. Water removal is performed with a vacuum that can be varied between 10 and $70 \mathrm{kPa}$. Vacuum levels are thus comparable with those used in real paper machine former sections. During sheet forming, in addition to the time evolution of vacuum, $P(t)$, the time evolution of the position of the pulp surface, $h(t)$,was measured with an ultra-sonic detector. SDS was used as the foaming agent for foam-laid structures, and for lowering the surface tension of the flowing medium, water, for water-laid structures. The average foam density was $300-350 \mathrm{~kg} / \mathrm{m}^{3}$ and the air content was thus $65-70 \%$. The foaming phase and forming device are described in detail in the articles (Lappalainen and Lehmonen 2012; Lehmonen et al. 2017). Foaming was started with a 51 batch of fiber-SDS-water solution. After foaming, the volume of foam was ca. 151 - the same as the volume of a fiber suspension batch before water forming. The forming consistency was $0.14 \%$ by weight and $0.45 \mathrm{~g} / 1$ by volume for foam-laid 


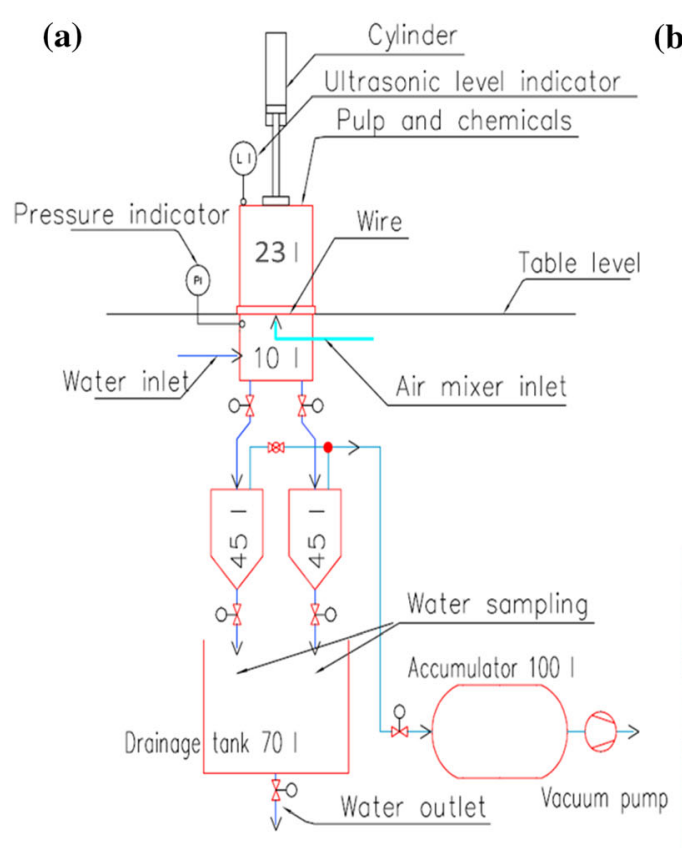

(b)
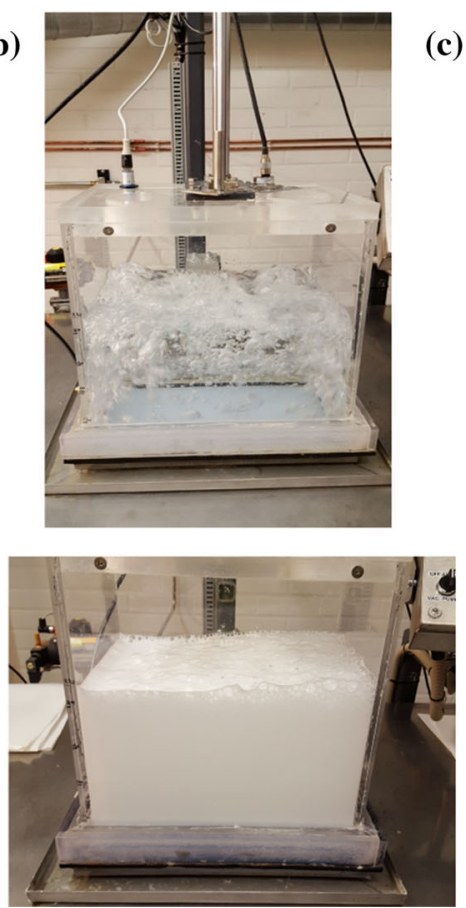

(c)
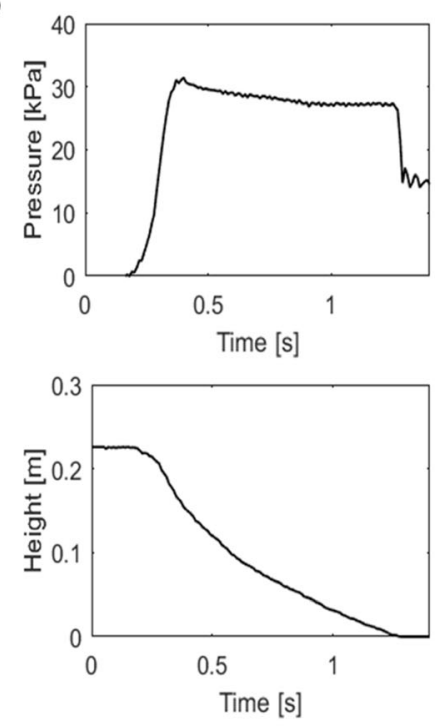

Fig. 4 a Diagram of the vacuum-assisted sheet former. b Photographs of the pulp chamber. In water forming the chamber is filled with a water suspension; in foam forming the

structures. Correspondingly, the forming consistency was $0.043 \%$ by weight and $0.44 \mathrm{~g} / \mathrm{l}$ by volume for water-laid structures. The target basis weight was $85 \mathrm{~g} / \mathrm{m}^{2}$.

The foams were generated by mixing the fiberSDS-water solutions for $5 \mathrm{~min}$ with a mechanical mixer with a mixing speed of $2200 \mathrm{rpm}$. The SDS dosage varied between 0 and $5 \mathrm{~g} / \mathrm{l}$. This foaming time was long enough to yield a stable foam with a homogeneous bubble size distribution having an average bubble diameter of $60 \mu \mathrm{m}$ (Lappalainen and Lehmonen 2012). After the foaming phase, the foamlaid suspension was poured into the acrylic mold and dewatered. Correspondingly, a 51 batch of water-laid suspension was poured into the acrylic mold and filled with the rest of the process water until the final volume was reached. Before the forming phase, the underside of the mold, having a volume of 10 , was filled with process water. The same procedure was carried out for both forming methods.

In forming with moderate and high vacuum levels, the time evolution of hydrodynamic resistance $R$ of a filtered sheet is given by the formula (Koponen et al. 2015) chamber is filled with a foamed suspension. c Examples of measured vacuum and surface level profiles during water forming

$R(t)=\frac{P(t)}{b(t) v(t)}$,

where $b$ is the basis weight of the sheet (expressed in SI units), and $P$ and $v$ are the vacuum level and the filtration speed in the filtration process. The flow resistance combines both the effect of the resistance due to the porous structure and the effect of fluid viscosity on the hydrodynamic friction during the filtration process. For simplicity, the small resistive effect of the supporting porous plate and wire have been omitted in Eq. (1), as their contribution to the overall resistance is small with the used forming device. The fluid velocity $v(t)$ in Eq. (1) can be calculated from the time derivative of the measured suspension surface level $h(t)$. As the sedimentation speed of fibers can be neglected, the instantaneous basis weight of the fiber sheet $b(t)$ is obtained from the equation

$b(t)=c[h(0)-h(t)]$,

where $c$ is the consistency of the suspension (expressed in $\mathrm{kg} / \mathrm{m}^{3}$ ). It was shown in Koponen et al. 
(2016a, b) that in water forming, due to elastic and plastic compression, the hydrodynamic resistance follows the formula

$R(t)=\beta P(t)+\alpha[I(t)-\Psi(t)]+\gamma$,

where $\alpha, \beta$ and $\gamma$ (zero-pressure resistance) are material parameters, $P$ is the vacuum level, $I(t)=$ $\int_{0}^{t} P \mathrm{~d} t$ is the impulse due to vacuum, and $\Psi(t)=$ $\frac{c}{b} \int_{0}^{t} I v d t^{\prime}$ is a correction term which takes into account the different impulse histories of the fiber layers of the filtered sheet. As the used furnish is rather stiff, and the filtration time is short, resistance is dominated here by the elasticity term $\beta P$.

Below, Eq. (1) is used to calculate the time evolution of the sheet resistance for both water forming and foam forming. Then, parameters $\alpha, \beta$ and $\gamma$ in Eq. (3) are solved for water forming with regression analysis. The sheet resistance during foam forming is compared with water forming by using Eq. (3) for water, and substituting the measured foam forming vacuum profile to it.

The set point levels for the vacuum accumulator were 30,50 and $70 \mathrm{kPa}$. In practice, the realized vacuum levels varied somewhat. The vacuum levels were on average $15 \%$ lower for foam than for water, probably due to higher pressure losses in the device and expansion of air below the wire.

Pressing and drying of sheets

Some formed samples were left unpressed, while others were wet pressed at levels of 50 and $350 \mathrm{kPa}$ according to ISO standard 5269:1. Unpressed sheets were dried with a cylinder drying device (Kodak rotary drum dryer) and the wet pressed sheets were dried on the plates. All paper samples were stored and analyzed in a standard air conditioned room at $\mathrm{RH}$ $50 \%$ and $23{ }^{\circ} \mathrm{C}$.

\section{Characterization of papers}

The basis weight of the paper samples was measured according to ISO standard 536:1995 and thickness according to ISO standard 534:1998. Density was determined based on the measured basis weight and sheet thickness. The tensile strength properties of the paper samples were measured with a Lloyd tensile tester in accordance with ISO standard 5270:1998.
The z-directional tensile strength of the paper samples was measured according to ISO standard 15754:2009. Ten parallel measurements were made when sheet properties were analyzed and the error bars in the figures show their standard deviation. Air permeability was measured with a Lorentzen \& Wettre Bendtsen Permeance SE 166 Tester according to ISO standard 5636-3:2013. Roughness was measured with a Lorentzen \& Wettre Bendtsen Roughness SE 164 Tester according to ISO standard 9791-2:2013. Compressive strength was measured with a Büchel K455 Short span compression tester in accordance with ISO standard 9895:2008.

The specific $\beta$-formation (Komppa and Komppa 1996) of the paper samples was measured by exposing a storage phosphor screen to $\beta$-radiation of Carbon-14 through the samples. The screen was scanned with a Fuji BAS-1800 II SPS reader. Then, the obtained radiation absorption map was converted into a basis weight map. The size of the scanned area was $100 \mathrm{~mm} \times 100 \mathrm{~mm}$ and the original scanning resolution was $100 \mu \mathrm{m}$. Finally, the resolution was transformed to Ambertech resolution $(1 \mathrm{~mm})$ and the specific $\beta$-formation values were calculated (i.e. the standard deviation of $\beta$-formation values were divided by the square root of the basis weight). Ambertech resolution $(1 \mathrm{~mm})$ is widely used in the industry instead of the original scanning resolution $100 \mu \mathrm{m}$; for this reason the resolution was transformed to Ambertech resolution.

The scattering coefficient of paper sheets is generally proportional to the specific surface area of the paper, and is thus inversely proportional to the total area of fiber bonds (Haselton 1954, 1955; Rennel 1969). Increasing the fiber web density, for example, decreases the specific surface area, leading to decreasing scattering coefficient. The scattering coefficient of sheets was measured with a Minolta Spectrophotometer CM-3610d.

\section{SEM imaging}

SEM imaging was carried out with a Merlin FE-SEM (Carl Zeiss NTS GmbH, Germany) with gold sputter coating at $20 \mathrm{~mA}$ for $30 \mathrm{~s}$ (Kouko et al. 2018). The sample was attached to an aluminum specimen stub with double-sided carbon adhesive tape, and the SEM imaging was performed using $3.0 \mathrm{keV}$ electron energy and using a secondary electron detector. The image 
size was $2.3 \mathrm{~mm} \times 1.7 \mathrm{~mm}(2048 \times 1536$ pixels $)$ and the pixel size was $1.1 \mu \mathrm{m}$.

\section{Results and discussion}

Forming of water-laid and foam-laid structures

The dewatering time in water and foam forming is presented in Fig. 5 as a function of the measured vacuum level. Dewatering starts when the pressure valve is opened and dewatering ends when the height of the water/foam column becomes zero. In water forming, the dewatering time was almost constant at all vacuum levels. This was expected, as hydrodynamic resistance (see Eq. 3) is dominated here by the vacuum. In foam forming, dewatering time decreased with increasing vacuum. At the lowest vacuum level dewatering time was on average 50\% higher in foam forming than in water forming, but with higher vacuum levels dewatering times of water and foam forming were roughly equal. This result is surprising as the viscosity of foam is significantly higher than the viscosity of water. We will elaborate on this interesting behavior of foam forming in detail below by studying the time development of the hydrodynamic resistance of the filtered sheet during water and foam forming.

Figure $6 \mathrm{a}, \mathrm{b}$ show examples of a measured vacuum profile and a measured column height in water forming. Figure $6 \mathrm{c}$, d show the corresponding drainage speed and hydrodynamic resistance of the sheet
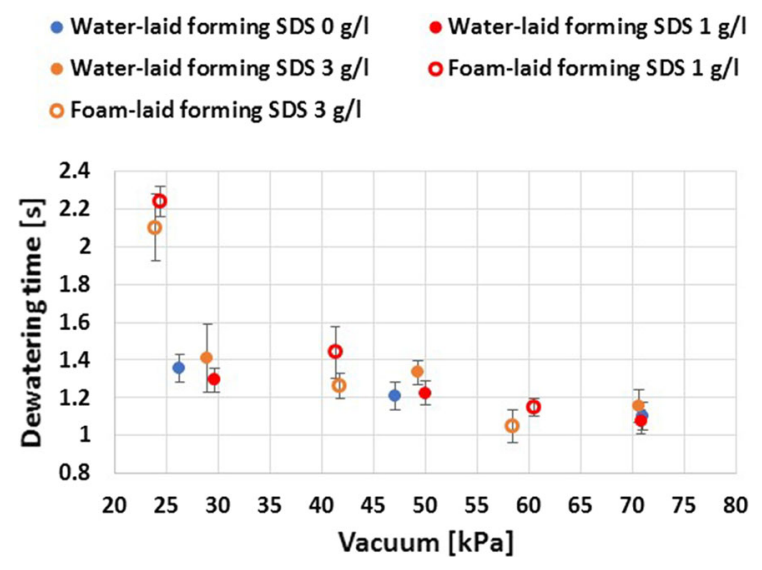

Fig. 5 Dewatering time as a function of vacuum level for water-laid and foam-laid forming as a function of time. In Fig. 6d the prediction of the viscoelastic model Eq. (3) is also shown. Here the model parameters $\beta=0.054, \alpha=0.038$, and $\gamma=1280$ $\left(\mathrm{r}^{2}=0.94\right.$, LAR robust fit in Matlab) were obtained by fitting the model simultaneously to all 39 water forming cases. Notice that hydrodynamic resistance is a bulk property of the filtered sheet. Thus, even though the hydrodynamic resistance remains almost constant after the vacuum level has saturated, the drainage speed gradually decreases due to the thickening of the filtered sheet.

Figure $7 \mathrm{a}-\mathrm{d}$ show the measured vacuum levels and the corresponding time evolution of the basis weight [calculated from Eq. (2)] for three water forming and foam forming cases. We see that $50 \%$ of the basis weight is filtered during the first $0.3 \mathrm{~s}$, after which the basis weight curves start to separate. The drainage rates for the three foam forming cases are shown in Fig. 8 . The rather high variation in measured velocity is probably due to the difficulty in detecting the foam surface with the ultrasound detector. In Fig. 8 the red lines show a fit of $v=a /(t-b)$ to the measurement points. These functions are used in Fig. 9 instead of the measured drainage rate for calculating the time evolution of the hydrodynamic resistance of foam. The resistance curves for water forming, also shown in Fig. 9, have been calculated from Eq. (3) using the measured vacuum profiles of foam forming. We see in Fig. 9 that in all three cases the resistance of foam is initially $35-50 \%$ below that of water. The resistance then increases with time. By the end of the forming process resistance is $100 \%$ higher for foam at the lowest vacuum level and almost 50\% higher at the two other vacuum levels. This behavior was observed with all the 34 foam forming trial points. The observed relatively low resistance of the foam-formed sheets is at first glance very surprising as the viscosity of foam is 1-2 orders of magnitude higher than for water. We will discuss next several factors that may together explain this behavior.

The average pore size of foam formed fiber sheets was studied in Koponen et al. (2017) with X-ray tomography. It was found that with porosity of $0.8-0.9$ the average pore size was $30-60 \mu \mathrm{m}$. The average size of pores is thus smaller or of the same size as the typical diameter of bubbles, $60 \mu \mathrm{m}$. The compression level of the fiber sheet is not known but if we assume that the sheet porosity during filtering is e.g. 0.8 we can make a rough estimate of the average shear rate 
Fig. 6 Examples of a measured vacuum, b measured suspension height, c surface velocity calculated from the measured suspension height, and $\mathbf{d}$ hydrodynamic resistance of the filtered sheet, calculated from Eq. (1), as a function of time in water forming with the set point vacuum of $70 \mathrm{kPa}$. In (d) the prediction of Eq. (3), fitted to all water forming data, is also shown. SDS dosage $1.0 \mathrm{~g} / 1$ (a)

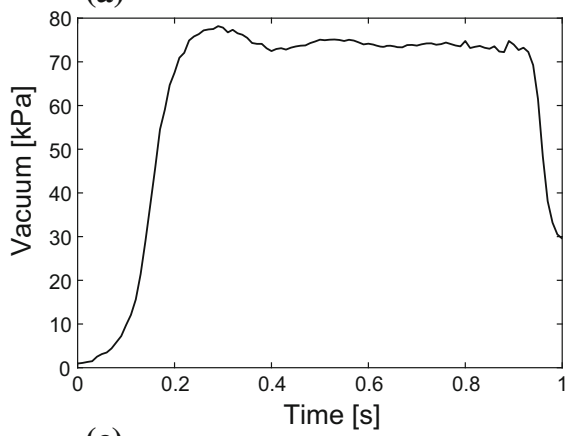

(c)

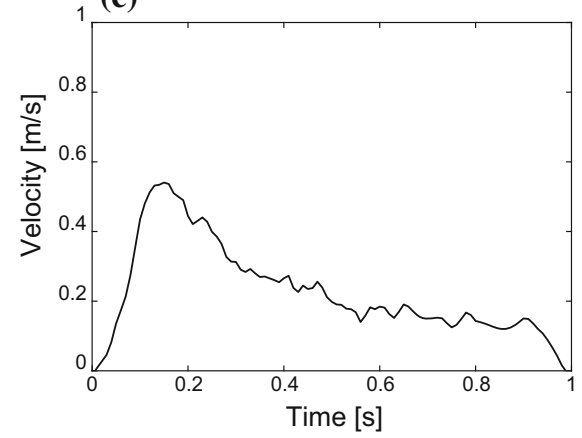

(a)

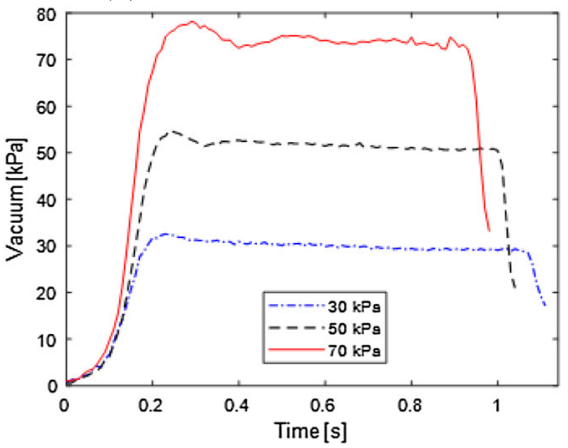

(c)

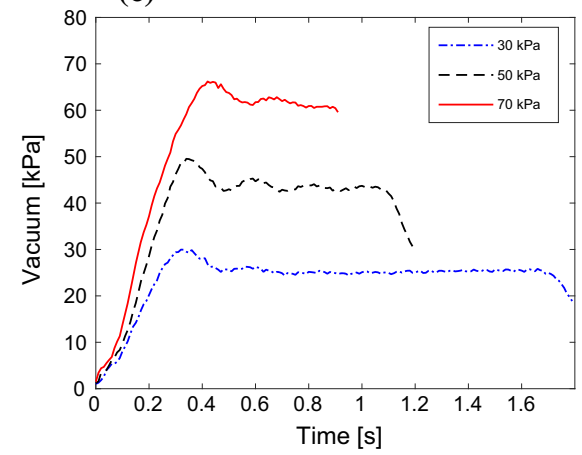

(b)
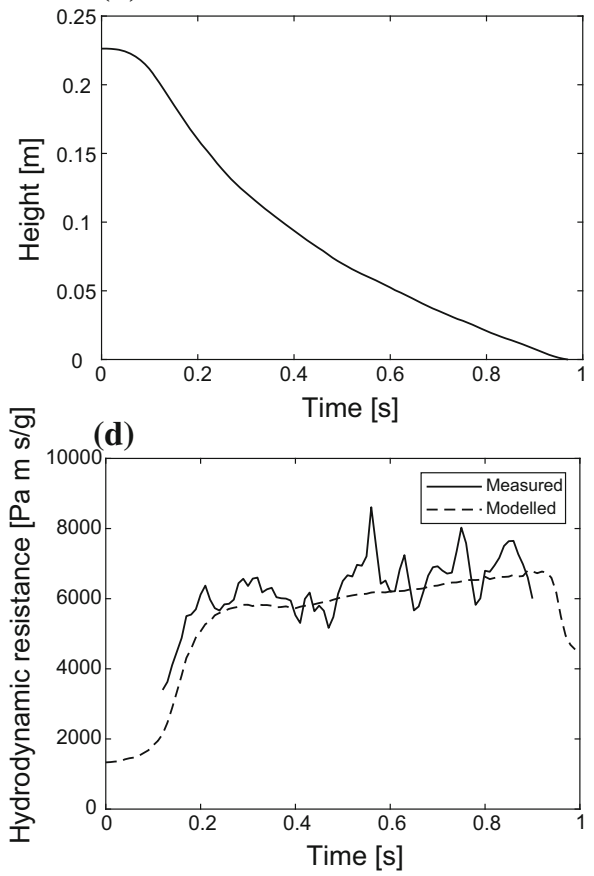

(b)

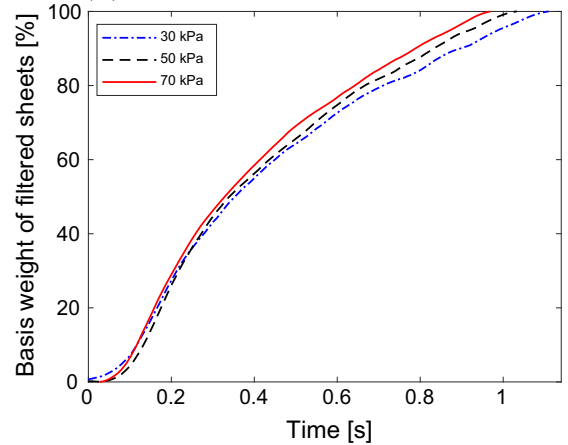

(d)

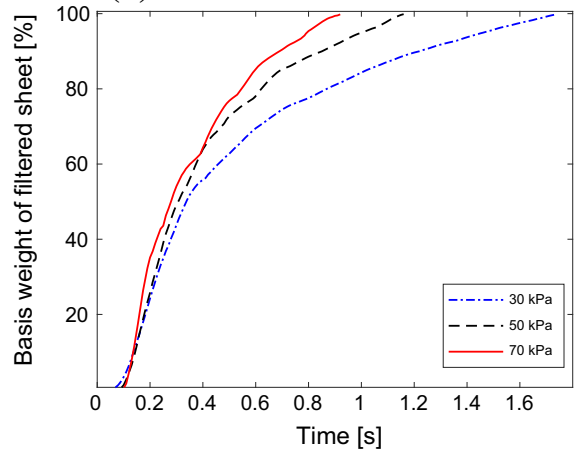



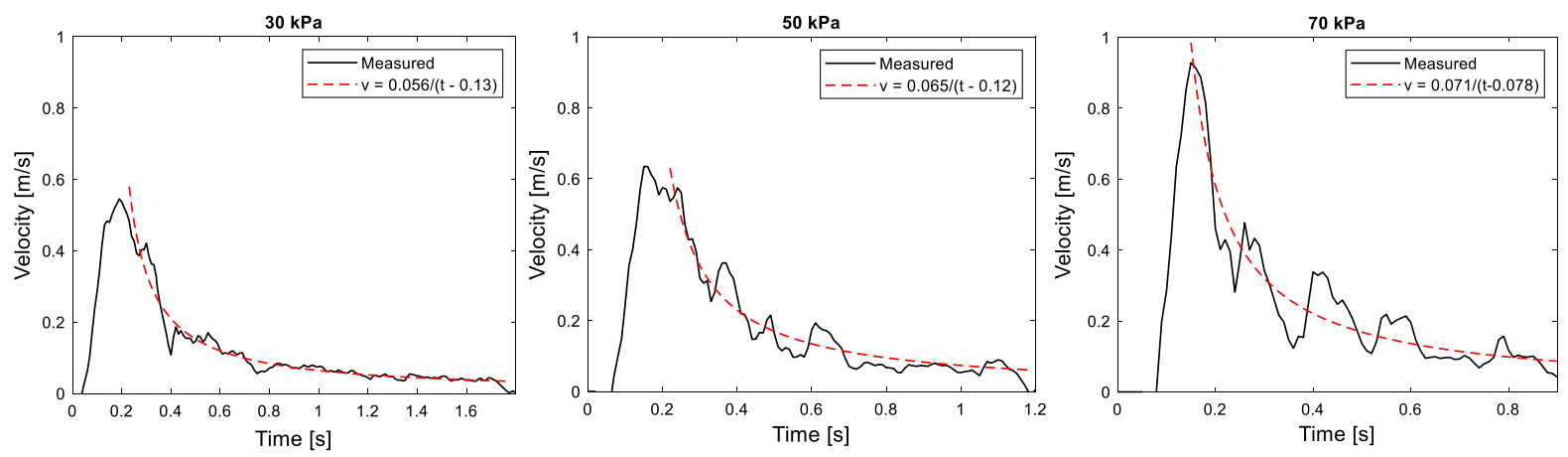

Fig. 8 Drainage rate for the foam forming cases shown in Fig. 7c, d. The red lines show a fit of $v=a /(t-b)$ to the measurement points following the velocity peak. SDS dosage $1.0 \mathrm{~g} / \mathrm{l}$

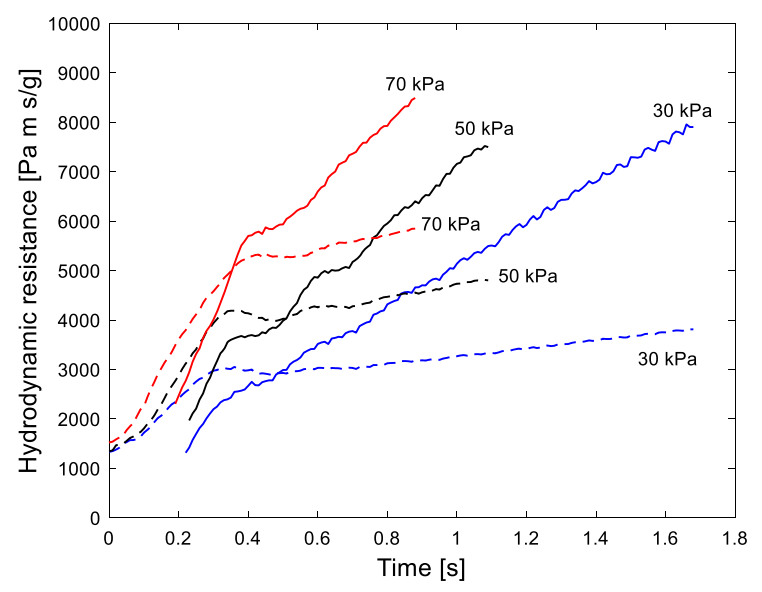

Fig. 9 Measured time evolution of the hydrodynamic resistance of the filtered sheets during foam forming (continuous lines), calculated from Eqs. (1) and (3). The corresponding curves for water forming are also shown (dashed lines). The numbers show the set point values of vacuum

$\gamma_{\text {ave }}$ during the filtering process. Assuming that the flow takes place in capillaries we get

$\gamma_{\mathrm{ave}} \sim \frac{2 v}{d \phi}$,

where $v$ is the velocity of the surface of the foam column, $d$ is the capillary diameter and $\phi$ is the sheet porosity. Table 1 shows with various surface velocities the estimated average share rate in the filtered sheet and the subsequent foam viscosity $\mu=0.98 \times$ $\gamma^{-0.6}$ taken from Fig. 1b. We see that with highest surface velocities the foam viscosities are indeed close to that of water due to strong shear thinning of the foam (Fig. 10).

The hydrodynamic resistance of a medium depends generally on two factors: the structure of the medium, and the viscosity of the fluid moving through it. It can be written in the form (Koponen et al. 2016a, b)

$R=\frac{\mu}{k \rho}$

where $\mu$ is viscosity, $k$ is sheet permeability, and $\rho$ is the density of the fibers in the filtered sheet. Foamformed sheets are often more bulky than corresponding water-formed sheets. It is possible that the bubbles support the fibrous structure, making it less dense also during forming. On the other hand, with similar density, the permeability of foam-formed sheets can be higher than water-formed sheets due to different pore structures. In Koponen et al. (2017), for example, foam-formed fiber sheets had 30-60\% higher permeability in the porosity region of $0.8-0.9$. Figure 10 shows a schematic of two possible cases of the flow of

Table 1 Estimated average shear rate calculated from Eq. (4) and subsequent foam viscosity with different velocities of the surface of the foam column

\begin{tabular}{llllllll}
\hline$v(\mathrm{~m} / \mathrm{s})$ & 1 & 0.5 & 0.25 & 0.1 & 0.05 & 0.025 & 0.01 \\
\hline$\gamma_{\text {ave }}(1 / \mathrm{s})$ & 83,000 & 42,000 & 21,000 & 8300 & 4200 & 2100 & 830 \\
$\mu_{\text {ave }}(\mathrm{mPa} \mathrm{s})$ & 1.1 & 1.7 & 2.5 & 4.4 & 6.6 & 10.0 & 17.3 \\
\hline
\end{tabular}


Fig. 10 Foam flow in a fibrous material is a combination effect of bulk foam viscosity and bubble distortion (Lemmen and Wassink 1990)
Flow through a wide capillary

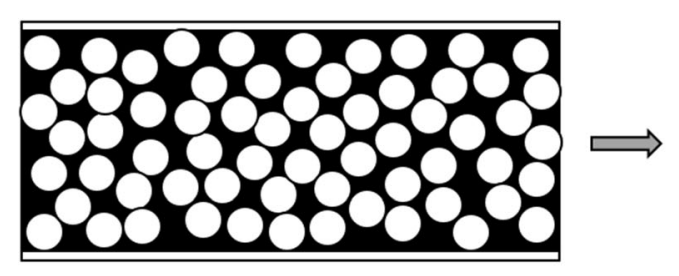

Flow through a constricted pore

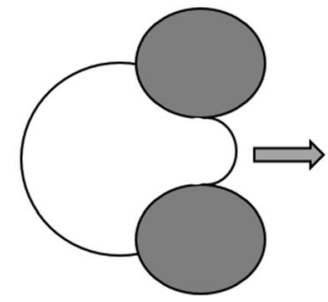

foam in a porous material. If the pores are clearly bigger than the typical bubble size, the foam behaves as a continuum fluid with a well-defined viscosity. On the other hand, if the bubbles are bigger than the pores the flow consists of the movement of individual bubbles and bubble deformations. Lemmen and Wassink (1990) studied the flow of foam (air content $83 \%$ ) through metal screens. They found that the measured pressure loss was too low by a factor two when compared with theoretical values calculated using the measured rheological behavior of foam. They concluded that foam flow must be described in terms of the combination effects of foam viscosity and bubble distortion. Hirasaki and Lawson (1985) found a decrease by almost a factor of 104 occurring in the apparent viscosity of foam (air content 83\%) in capillary tubes as the ratio of bubble radius to tube radius, , increased from 0.1 to 10 . When was 10 they reported apparent foam viscosities as much as $60 \%$ below that of water. As discussed above, Koponen et al. (2017) found with X-ray tomography that at a porosity of 0.8 , for example, the average pore diameter of a fiber sheet was $30 \mu \mathrm{m}$, which is clearly smaller than the typical diameter of bubbles. The porosity of the filtered sheet is here unknown during forming, but it seems probable that the constricted pore type flow of foam contributes to the observed low resistance of foam.

We can conclude that the observed low values of resistance of foam during forming (even below that of water) are probably caused by many effects such as structural differences in the filtered sheets, strong shear thinning of foams, and the low apparent viscosity of foam when the bubble size is bigger than the pore size of the filtered sheet. Still another possible explanation is the phase separation of air and water during flow; the viscosity of air and water are significantly below that of foam. The substantial increase in resistance with time, which is not seen in

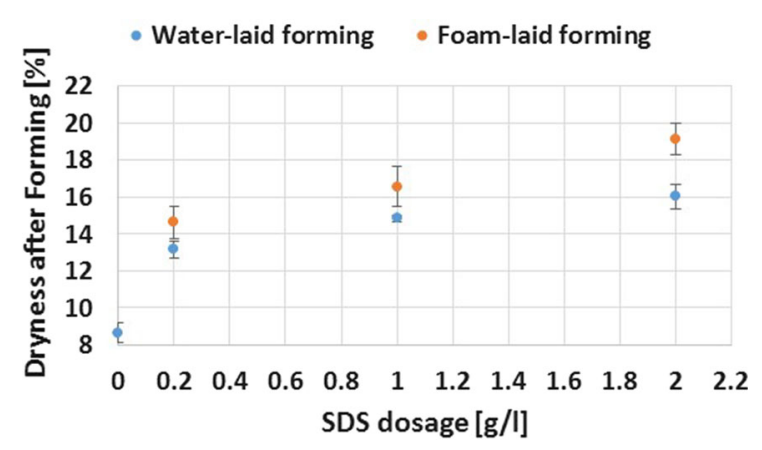

Fig. 11 Dryness level after the forming phase at the set point vacuum of $30 \mathrm{kPa}$

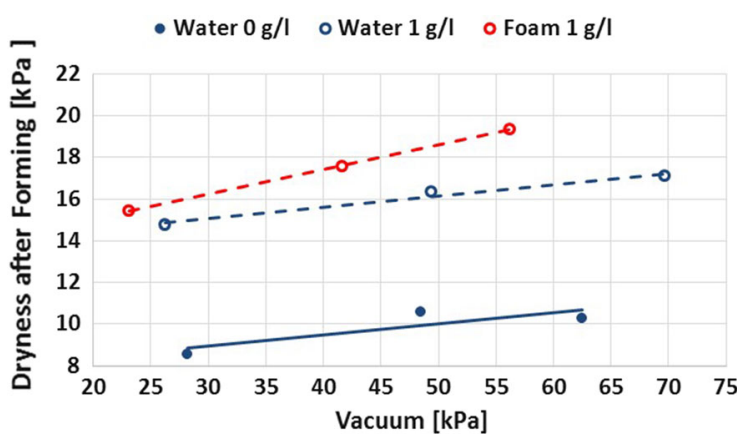

Fig. 12 Dryness after forming phase with different vacuum levels. Water forming was performed both with and without SDS. Vacuums for foam forming were estimated based on the vacuums of water forming as the pressure measurement was dysfunctional during this experiment

water forming, is likely related to shear thinning type behavior of the apparent viscosity of foam.

Dryness of wet sheets after forming and pressing

At the end of the forming, when the last remaining free water/foam goes through the upper surface of the filtered sheet, the amount of water inside the sheet depends on the structure and thickness of the sheet. 


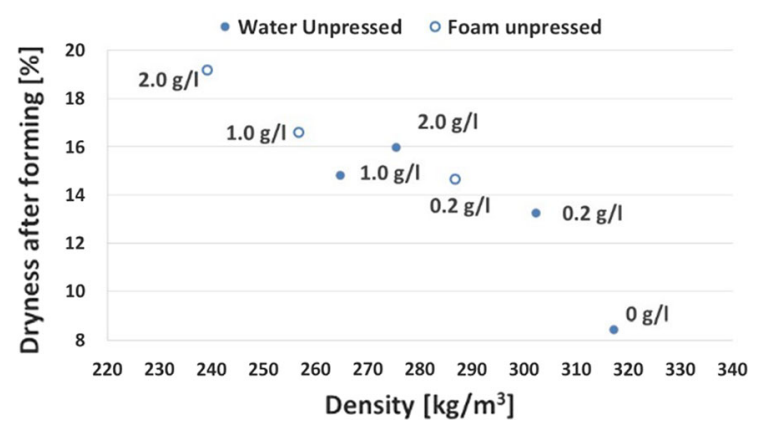

Fig. 13 Dryness after forming as a function of density and SDS dosage for unpressed dry sheets. The setpoint vacuum is $30 \mathrm{kPa}$

This part of the process is called compression dewatering, which is dictated by the pressure difference over the sheet. The second phase of the consolidation process is called displacement dewatering, where the free water is replaced by air. In this phase, capillary forces have an important effect as they impede dewatering (Aslund and Vomhoff 2008).

The dryness of the water-formed and foam-formed sheets is shown in Fig. 11 as a function of SDS dosage. Dryness is seen to increase significantly as SDS dosage increases. Foam forming gives consistently higher dryness, and the difference increases with increasing SDS dosage. Without SDS, dryness in water forming is $35 \%$ lower than with a SDS dosage of $0.2 \mathrm{~g} / \mathrm{l}$. This is clearly due to the SDS decreasing the surface tension of the water. The effect of SDS on dryness at dosages of 1.0 to $2.0 \mathrm{~g} / \mathrm{l}$, however, is probably due to decreasing dynamic surface tension. This is indicated by the fact that increasing the SDS dosage from 1.0 to $2.0 \mathrm{~g} / \mathrm{l}$ increased the dryness, although the static surface tension is the same in both cases (see Fig. 2).

Figure 12 shows the dryness after the forming phase at different vacuum levels for water and foam forming. Water forming was performed both with and without SDS. In both cases dryness increases with increasing vacuum level and the effect is slightly stronger in foam forming. Figure 13 shows the dryness after forming as a function of dry sheet density, and SDS dosage for unpressed sheets. We can see that higher dryness and lower density are obtained after water and foam forming for the final dry sheet with increasing SDS dosage.

As seen from Figs. 11, 12 and 13, foam forming gave consistently higher dryness than water forming also when SDS was used in water forming.

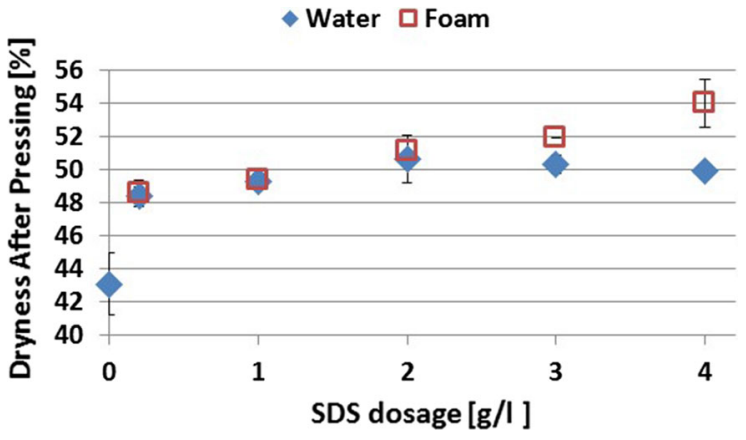

Fig. 14 Average dryness levels after wet pressing for waterlaid and foam-laid sheets as a function of SDS dosage. The sheets were formed with a $30 \mathrm{kPa}$ set point vacuum and the wet pressing pressure was $350 \mathrm{kPa}$

Additionally, foam forming was found to give lower density (higher bulk) for unpressed sheets. Higher dryness after the forming section has also been seen with foam forming at the pilot scale (Koponen et al. 2016a, b, 2018).

The superior dryness of foam forming after forming phase may be attributed to several possible factors. Firstly, when air starts to flow through the sheet at the end of forming, there is less water to remove from the sheet in foam forming. Secondly, air bubbles are known to remove water better from the sheet than pure air flow (Skelton 1987). This may be related to foam sealing the sheet (improving compression dewatering) and eliminating channeling of air flow through the sheet (improving displacement dewatering). Displacement dewatering may also be improved in foam forming due to bubble surfaces wiping the droplets away from fiber surfaces. Sheet permeability may also be higher in foam forming due to a higher number of large pores (Hjelt et al. 2011; Koponen et al. 2017) and

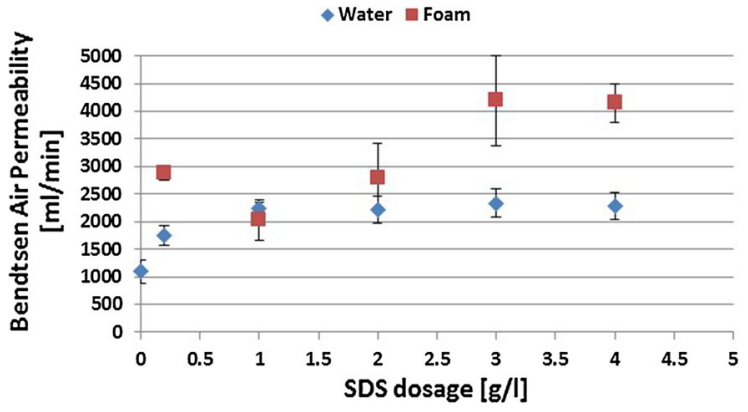

Fig. 15 Bendtsen air permeability of water-formed and foamformed sheets at different SDS dosages. Wet pressing pressure is $350 \mathrm{kPa}$ 
Fig. 16 Specific $\beta$ formation as a function of SDS dosage. The set point vacuum is $30 \mathrm{kPa}$ and wet pressing pressure is $0 \mathrm{kPa}$

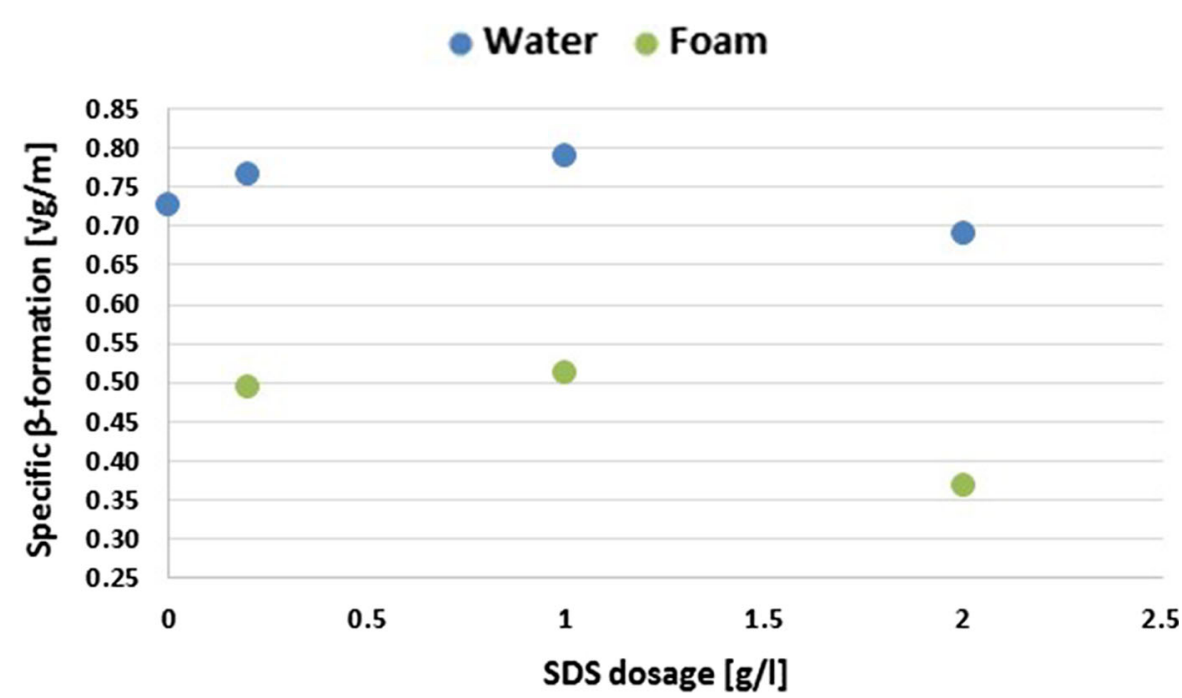

lower density. This would lead to higher flow rate of air, which improves displacement dewatering.

Figure 14 shows the dryness of water-formed and foam-formed sheets after wet pressing as a function of SDS dosage. Dryness is similar for both water-formed and foam-formed sheets up to $2.0 \mathrm{~g} / \mathrm{l}$ SDS dosage, despite foam having higher dryness after the forming phase (see Figs. 11, 12). With higher SDS dosage dryness of water-formed sheets decreases slightly (this might be related to the critical micelle concentration), whereas the dryness of foam-formed sheets keeps increasing. The dryness of water-formed sheets seems to depend on the surface tension of the water (see Fig. 3) - there is an excellent inverse correlation between them. In addition to surface tension, the observed improved dryness behavior of foam with increasing SDS dosage may be related to higher permeability of the sheet due to lower density before

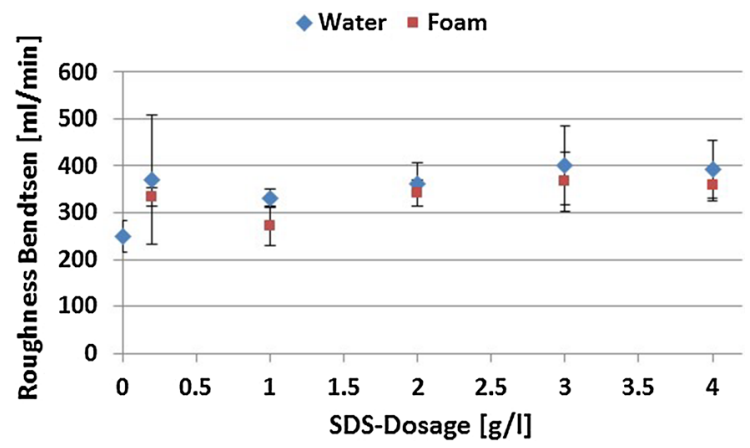

Fig. 17 Roughness as a function of SDS dosage. Wet pressing pressure is $350 \mathrm{kPa}$ pressing (see Fig. 13) and a more open pore structure due to a higher number of large pores (Hjelt et al. 2011; Koponen et al. 2017). The air permeability of the final dry sheets, shown in Fig. 15, correlates rather well with the dryness results shown in Fig. 14. Notably, foam forming has been found to improve dryness after wet pressing also at the pilot scale (Torvinen et al. 2015).

Properties of dry sheets

\section{Structural properties}

The evenness of material distribution in the in-plane direction was measured using the $\beta$-formation method. Figure 16 shows the $\beta$-formation of foam- and waterformed sheets as a function of SDS dosage. Foam forming achieved much better formation (i.e. lower $\beta$ formation value) even though the consistency in water forming was very low and lower than in foam forming. This suggests that the presence of the bubbles in the foam keeps the fibers separated in the suspension and prevents flocculation of the fibers during the drainage phase. The amount of surfactant seems to also have some effect on formation. The number of trial points is too low for any definitive conclusions, but formation seems to be clearly improved at the highest SDS dosage in the case of foam forming. The reason for this behavior could be that at the highest SDS dosage the bubbles are strong and stable and thus effectively reduce the agglomeration of fibers, thereby improving 
Fig. 18 Density as a function of SDS dosage at different wet pressing levels (dotted lines are guide for the eye)

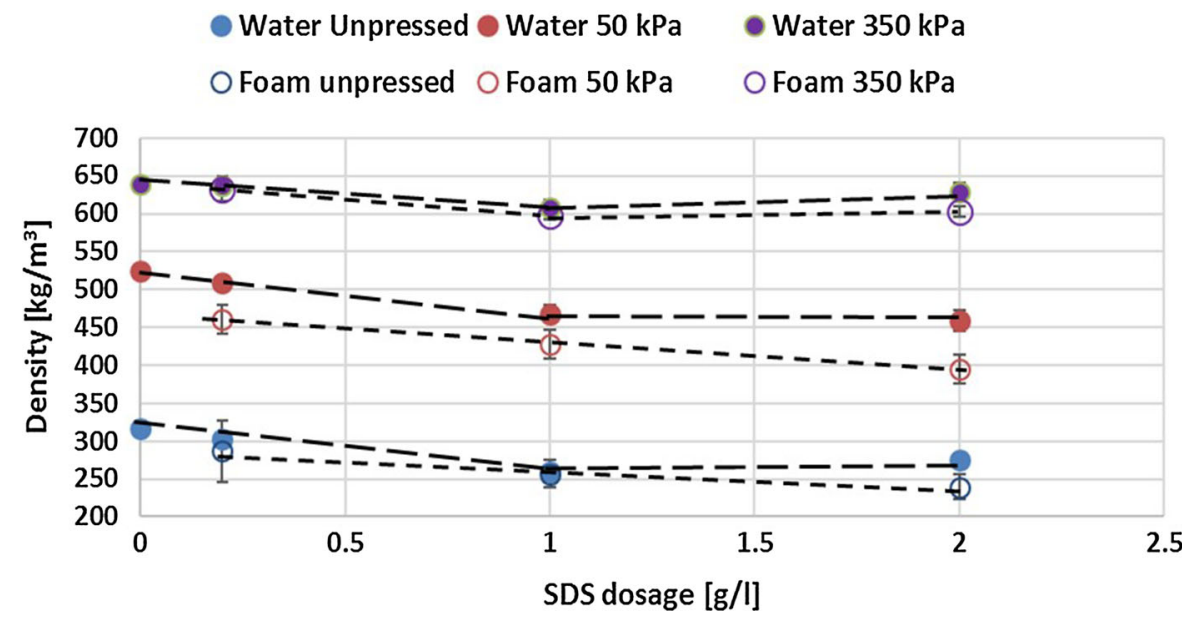

formation. Sheet roughness (Fig. 17) was also found to be affected by SDS dosage. The foam-formed sheets tended to have slightly lower Bendtsen roughness at all SDS dosages, but the difference was rather small (see Fig. 17). This might be due to the better formation and thus due to smoother surfaces of the foam-formed papers.

The formed papers were wet pressed at 0,50 and $350 \mathrm{kPa}$. As expected, wet pressing increased the density of the sheets considerably (see Fig. 18). Density decreased with increasing SDS dosage in both water and foam forming. With equal SDS dosage, foam forming typically gave slightly lower density. The density of the foam-formed sheets decreased linearly with increasing SDS dosage with the wet pressing pressures of 0 and $50 \mathrm{kPa}$. This behavior may be due to variation in foam strength. With the wet pressing pressure of $350 \mathrm{kPa}$ the curves for water and foam formed sheers were similar. The ability of foam to deform and compress and spring back and expand without bubbles breaking may therefore improve with increasing SDS dosage. In water forming, the density decreased linearly up to an SDS dosage of $1.0 \mathrm{~g} / \mathrm{l}$, upon which it more or less reached saturation. This behavior seems to correlate well with the surface tension of the solution (see Fig. 3). Indeed, Lyne and Gallay (1954) have shown that higher water surface tension draws the fiber surfaces closer to each other, leading to better inter-fiber bonding and thus stronger structures.

The results of Lindqvist et al. (2009) with waterlaid sheets and non-ionic surfactant have shown that surfactant concentrations, especially higher than the micelle concentration, also reduce wet adhesion between fibers and initial wet strength. This may increase detachment and debonding due to springback in the end of wet pressing. Here the surfactant is anionic and density decreases already at concentrations well below the critical micelle concentration. It is possible that SDS molecules attach to chemical components on the fiber surface, weakening adhesion between fibers at all SDS dosages.

The effect of SDS dosage on air permeability is shown in Fig. 15. Foam forming seemed to generally give significantly higher permeabilities. This is probably due to foam forming giving lower sheet density (air permeability seems to correlate rather well with sheet density, see Fig. 18) and having a more open pore structure. In earlier research, tomographic images of foam-formed sheets explained the higher permeability also when sheet densities were similar (Koponen et al. 2017). This was found to be due to differences in pore structure-foam-formed sheets were found to have a greater number of large pores, the size of which was of the order of the foam bubbles. Similar conclusions were also drawn by Hjelt et al. (2011).

The paper structure at the fiber scale was analyzed by SEM imaging (Figs. 19, 20, 21). Although no large differences in paper structure were observed, the foam-formed sheets appeared to have a slightly more open structure and the presence of pores could also be seen. In addition, some indicative differences in the number of surface fibrils and fibrillar bridges between adjacent fibers were observed. Based on the SEM images, fibrils seemed to be more collapsed onto the 

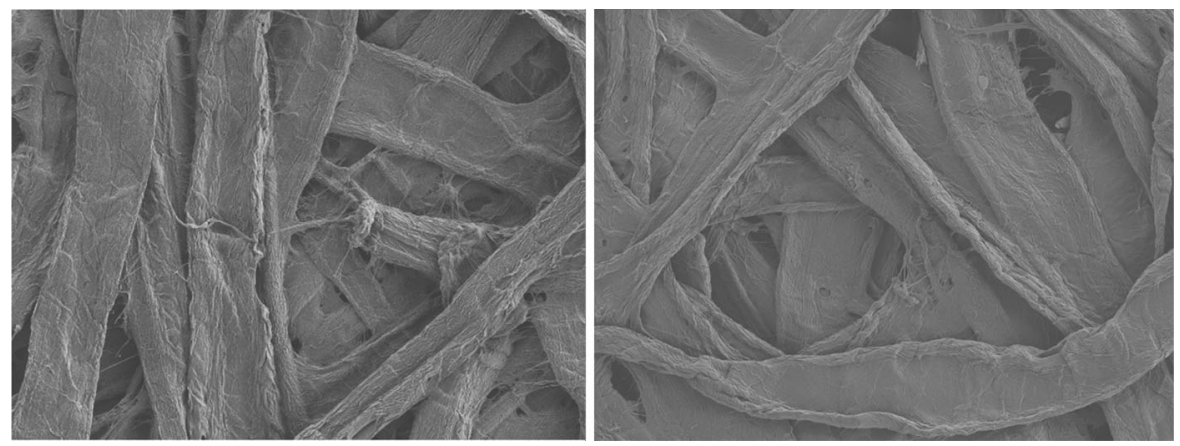

Fig. 19 Water-laid structure (left) versus foam-laid structure (right) at SDS dosage $0.2 \mathrm{~g} / \mathrm{l}$. The set point vacuum is $30 \mathrm{kPa}$ and wet pressing pressure is $350 \mathrm{kPa}$
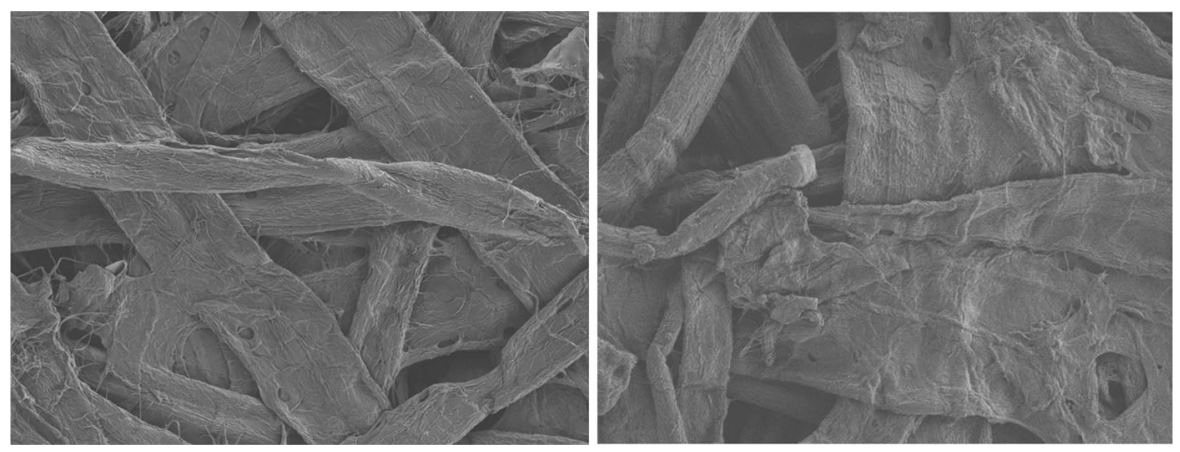

Fig. 20 Water-laid structure (left) versus foam-laid structure (right) at SDS dosage $1.0 \mathrm{~g} / \mathrm{l}$. The set point vacuum is $30 \mathrm{kPa}$ and wet pressing pressure is $350 \mathrm{kPa}$
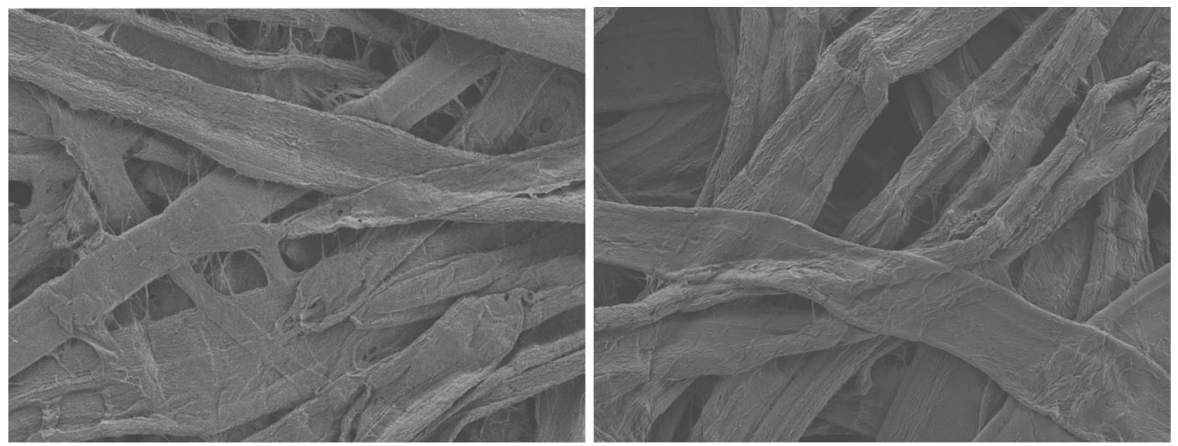

Fig. 21 Water-laid structure (left) versus foam-laid structure (right) at SDS dosage 2.0. The set point vacuum is $30 \mathrm{kPa}$ and wet pressing pressure is $350 \mathrm{kPa}$

fiber surfaces in the foam-laid papers than in waterlaid papers, where also more fibrillar interfiber bridges could be seen. These fibrillar bridges may contribute not only to interfiber bonding, but also to the light scattering properties of the paper. Generally, the absence of fibrillar interfiber bridges in foam-formed papers may be an indication of the effect of air bubbles separating fibers and also preventing the formation of interfiber fibrillar bridges. The difference between water- and foam-formed papers was more evident at the highest SDS dosage level. This behavior could be attributable to the lower surface tension with increasing SDS dosage reducing the capillary forces that collapse the fibrils onto the "parent fiber" in the waterlaid network, and the more stable bubbles reducing the mobility of the fibrils in the foam-laid fiber network. 


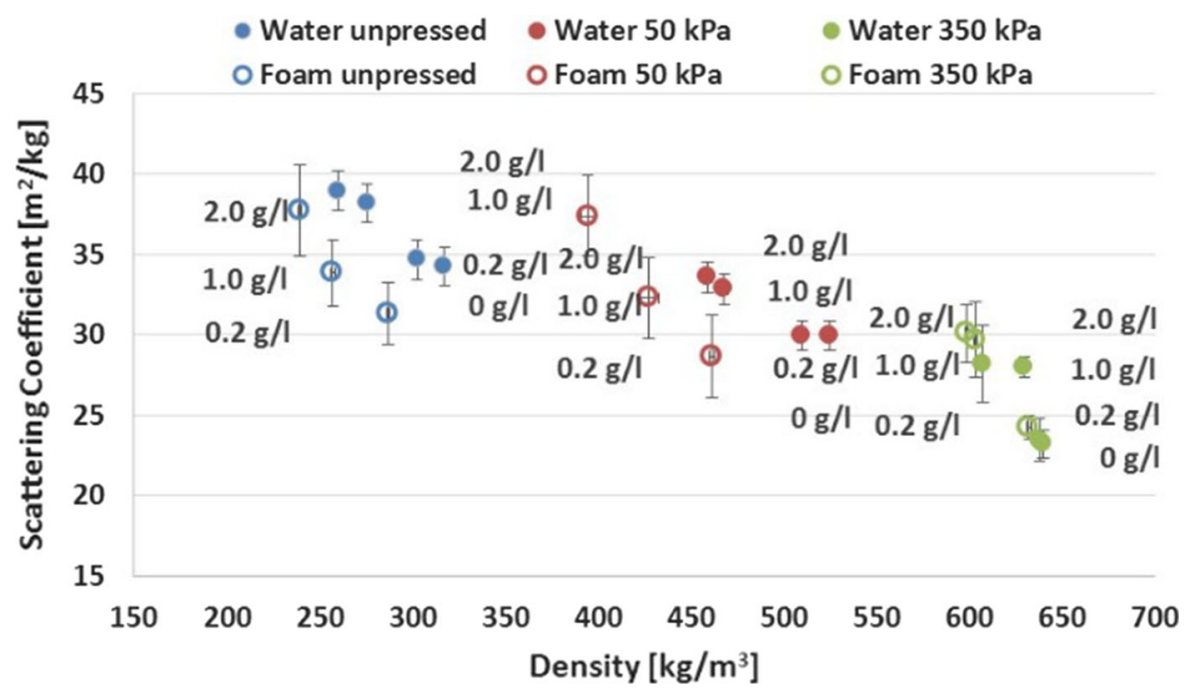

Fig. 22 Scattering coefficient of water-laid and foam-laid papers as a function of density. SDS dosage is also shown

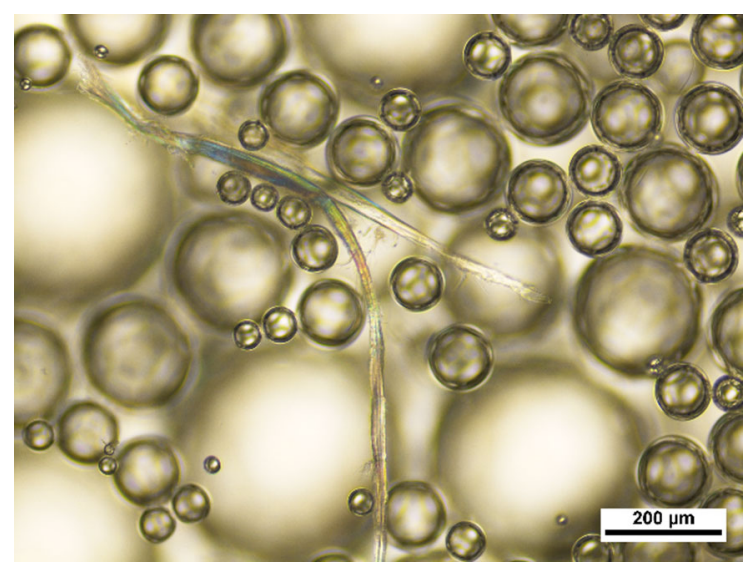

Fig. 23 Bubbles may push fibers into contact

Anionic surfactant, if adsorbed to the fibril surfaces, may also increase the electrostatic repulsion forces and prevent the collapse of surface fibrils.

\section{Interfiber contact and z-strength}

Density is among the most important basic properties of paper sheets. Density is strongly related to the structure and strength of paper as it correlates well with the number of contacts between fibers. Within a certain range the density correlates linearly with the relative bonded area (RBA) (Retulainen and Ebeling 1993). The amount of interfiber contact is reflected in the light scattering coefficient, as it is a measure of the amount of air-fiber interphases and is linearly related to the unbonded area of the sheet (Rennel 1969). Increased interfiber bonding therefore results in a decreased light scattering coefficient.

Scattering coefficient and density generally have a linear relationship. This was the case also in the present study when the SDS dosage was varied. However, the relationship was different for different wet pressing levels (see Fig. 22). Additionally, at a certain density the light scattering coefficient was 3-5 units lower for the foam-formed sheets than for the water laid sheets, except at the highest wet pressing level where no clear difference could be seen. This difference may be an indication of a higher interfiber contact area in foam-formed sheets. This may be due to a structural effect caused by the foam bubbles. The foam bubbles limit the possible locations of the fibers, resulting in more open pores but also in more fiber contacts at the areas in-between the bubbles (see Fig. 23). An indication of this mechanism was seen also in Koponen et al. (2017) where the specific surface area of non-pressed water and foam-formed sheets was determined from tomographic images. It was found that the specific surface area of foamformed sheets was systematically $10 \%$ smaller for both bleached kraft pulp and CTMP sheets.

However, there is also another possible contributing factor to the difference in light scattering. The earlier assumed higher number of fibrillar bridges between fibers in water-laid sheets may contribute to this difference. The fibrillar bridges scatter light, but 
Fig. 24 Effect on z-directional strength of density varied by wet pressing pressure, SDS dosage and introduction of foam to the fiber suspension. The lines are guide for the eye

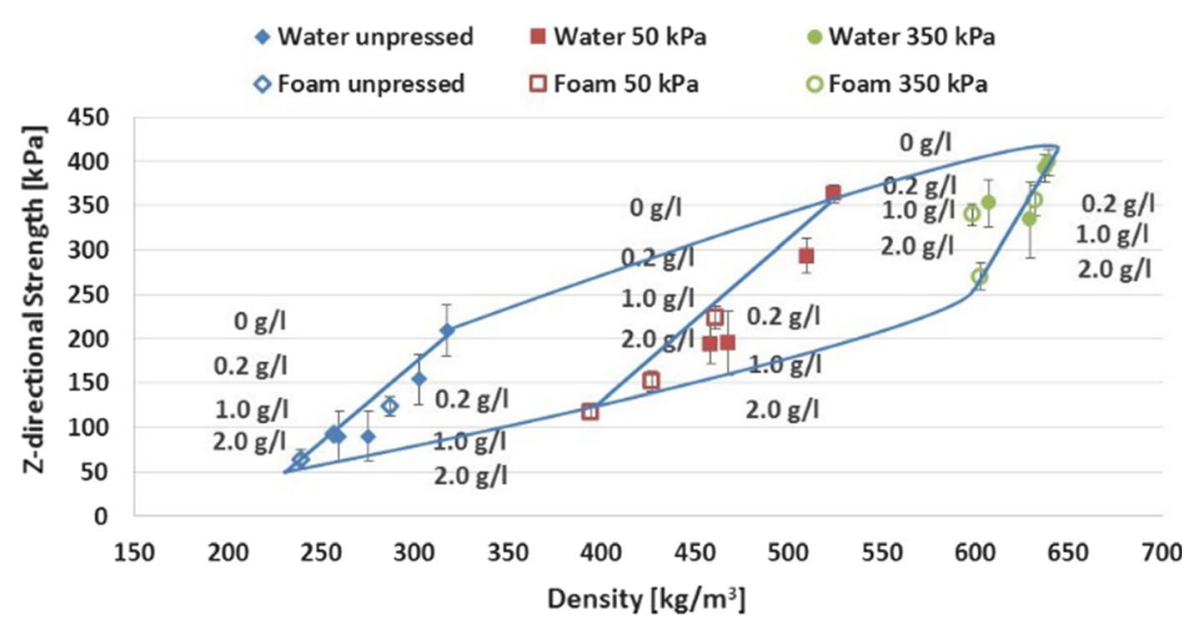

may also contribute to interfiber bonding. It is evident that in foam forming no fibrillar bridges can be formed through bubbles. At the highest wet pressing level applied here there were no differences between foamand water-formed sheets (see Fig. 22). This suggests that wet pressing pressure has an overriding effect on surface tension forces in bringing fibers together and determining the sheet density and interfiber contacts.

The bonding strength of paper can be measured by a z-directional strength test (see Fig. 24). The increased density brought about by wet pressing shows that greater interfiber contact increases z-strength. However, an increasing amount of SDS decreases the z-strength very strongly, and the presence of foam also has a decreasing effect. The slope with density is much steeper than with wet pressing, which suggests that the decrease is not only due to reduced fiber contact area, but also due to weaker bonds (and lower specific bond strength). However, with the highest wet pressing pressure the effect of SDS and the introduction of foam is much smaller than with unpressed samples, suggesting that the role of surface tension forces or bubbles is overridden by the wet pressing pressure. This may, once again, indicate that wet pressing pressure is the dominating factor in bringing fibers into contact.

Normally in water forming no SDS is used. When analyzing the cases (in Figs. 22, 24) in which water forming was used without SDS $(0 \mathrm{~g} / \mathrm{l})$ and foam forming at an SDS dosage of $2 \mathrm{~g} / \mathrm{l}$, the foam-formed
Fig. 25 Tensile strength of water-laid and foam-laid papers, as a function of density. SDS dosage is also shown

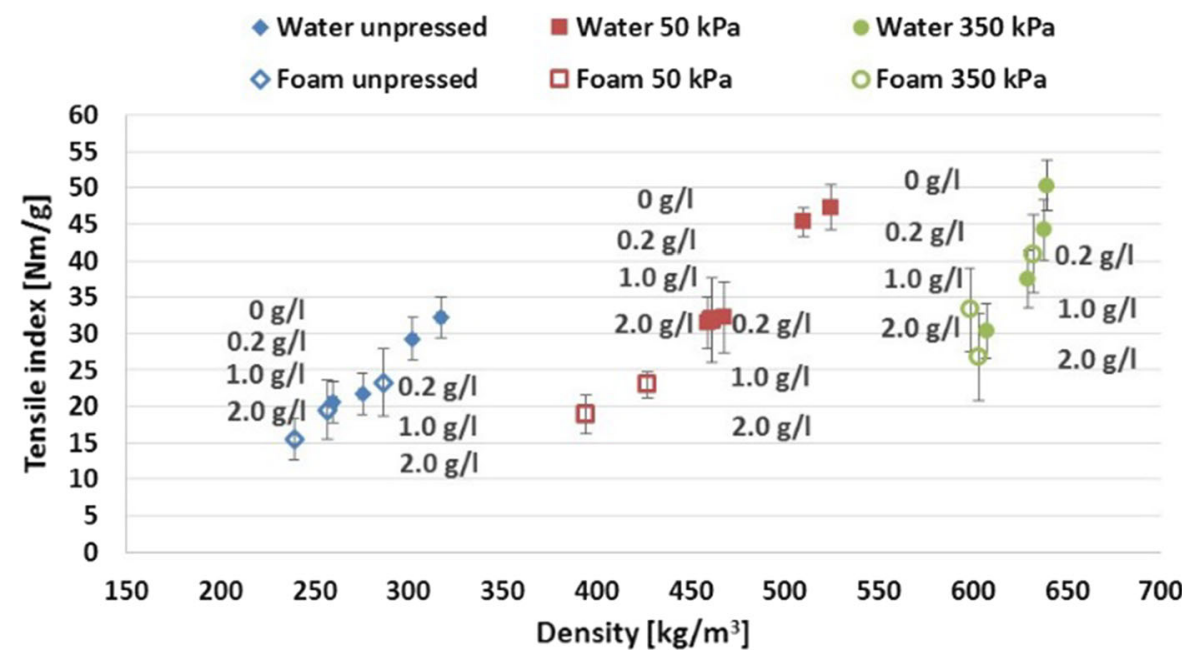


Fig. 26 Strain at break of water-laid and foam-laid papers as a function of density. SDS dosage is also shown

Fig. 27 Modulus of elasticity of water-laid and foam-laid papers as a function of density. SDS dosage is also shown
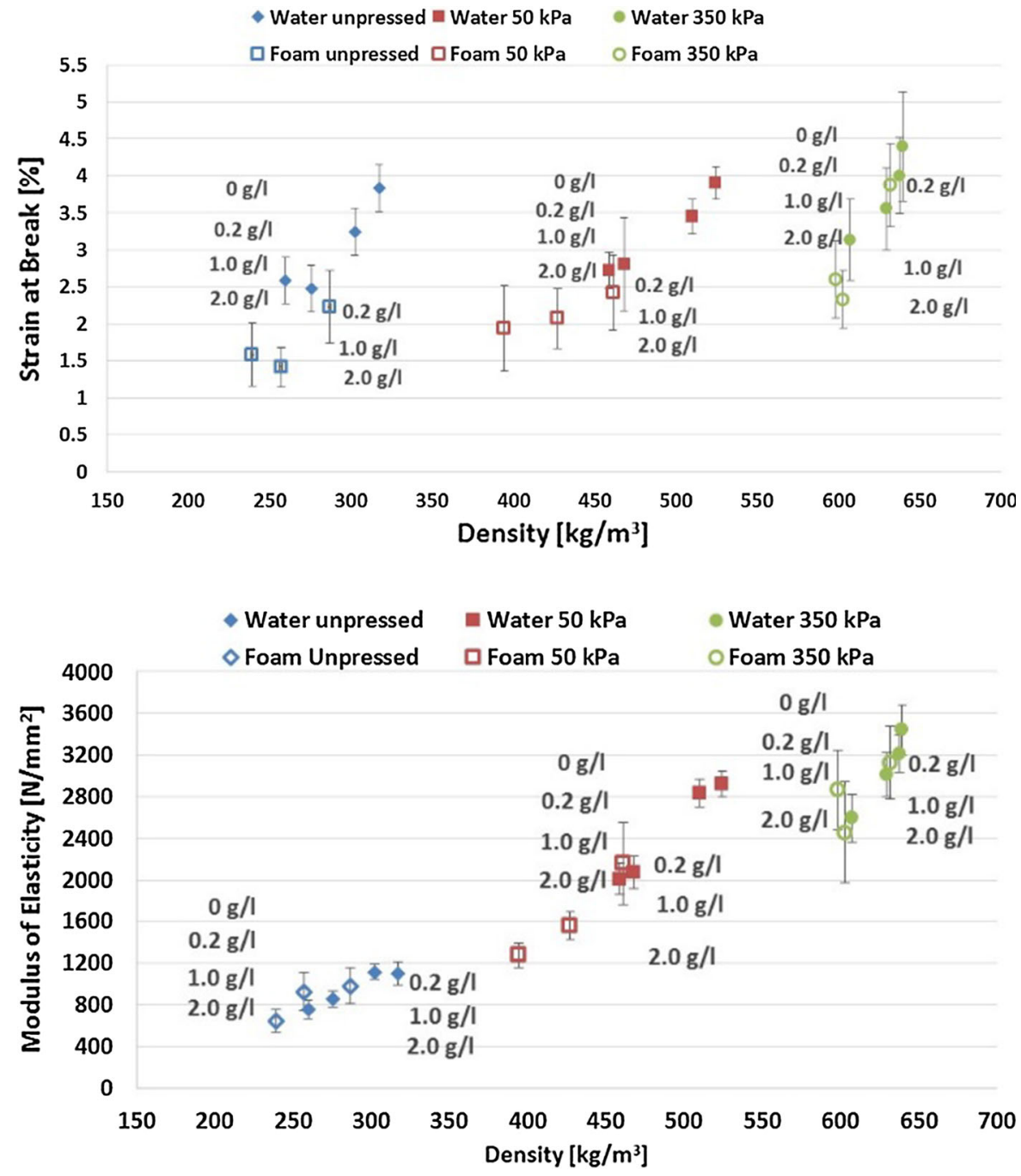

sheets had a higher light scattering coefficient and lower density and a lower number and strength of interfiber bonds.

\section{In-plane strength properties}

Tensile strength, strain at break, and elastic modulus are presented in Figs. 25, 26 and 27. They are depicted as a function of sheet density, which in turn is a measure of the amount of interfiber contacts. The drying shrinkage of the sheet was prevented and shrinkage or shrinkage stress should not have an effect on the strain at break. Figures 25, 26 and 27 very closely resemble Fig. 24, indicating that tensile strength and strain are mainly determined by interfiber bonding. Tensile strength and strain at break depend on the strength of the interfiber bonding. The increase of the strain is better explained by the strength of the bonds. On the other hand, the elastic modulusbecause the measurement does not involve disruptive testing-is in principle not dependent on the dry strength of bonds. Therefore the density-elastic modulus relationship shows an overall linearity. With the over-all linearity we mean that the effects of reducing SDS dosage, and increasing wet pressing pressure are nearly linear and the slopes of the lines are very similar. The difference in the slopes is much smaller than in the case of tensile strength or strain at break 


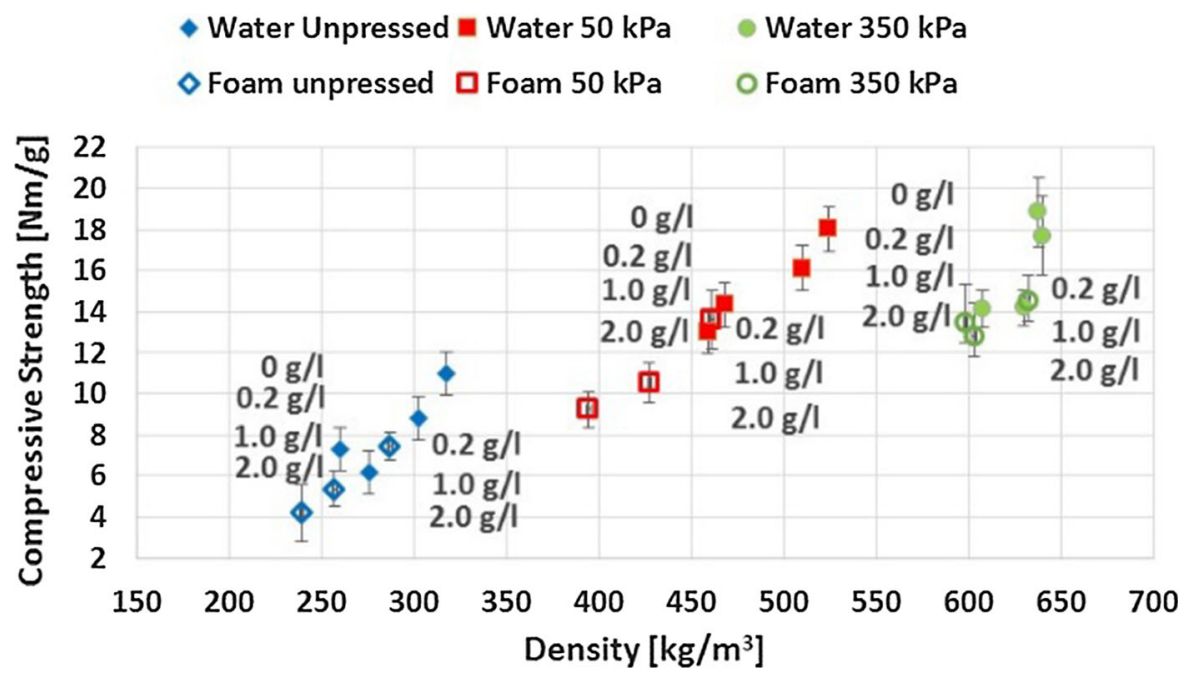

Fig. 28 Compressive strength (SCT) of water-laid and foam-laid papers as a function of density. SDS dosage is also shown

because the strength of the bonds does not have an effect on elastic modulus. The remaining difference may be explained by different fibre segment activation due to different drying stresses. However, there is a certain deviation from the general overall linearity that can be attributed to the effect of the SDS on shrinkage stress, which has a crucial role in the load bearing capacity of the network (cf. Lobben 1976). It can be speculated that in the wet sheet SDS reduces "adhesion before shrinkage" (Lindqvist et al. 2009; Page and Tydeman 1966); therefore, the drying shrinkage of the fibers is not transmitted so strongly to the whole network and the load bearing capacity is lower.

\section{Compressive strength}

The compressive strength test results (see Fig. 28) showed similar behavior to in-plane tensile strength and, especially, modulus of elasticity. Based on the current results, the compressive strength of water- and foam-formed sheets seemed to be at the same level at the highest densities and highest surfactant dosage levels.

\section{Conclusions}

The use of aqueous foams as a carrier fluid for pulp fibers was studied at the laboratory scale. The waterlaid and foam-laid papers were formed using a dynamic vacuum-assisted sheet former which simulates the dynamic dewatering phase of the forming section.

Although foams are much more viscous than water, the dewatering times were approximately equal in water and foam forming at higher vacuum levels - at the lowest vacuum level the dewatering time of foam was $50 \%$ higher. The dynamics of the forming phase was different in water and foam forming. The hydrodynamic resistance of sheets was approximately constant during water forming. In foam forming resistance was initially smaller than in water forming but it increased with time, being higher at the end of the forming process. The low resistance during foam forming is very surprising as the viscosity of foam is 1-2 orders of magnitude higher than water. The observed low values of resistance during foam forming were probably caused both by structural differences in the filtered sheet, strong shear thinning of foam, and a low apparent viscosity of foam when the bubble size is bigger than the pore size of the sheet. The significant increase in resistance with time, which is not seen in water forming, is probably related to the shear thinning type behavior attributable to the apparent viscosity of the foam.

Inclusion of SDS improved the dryness of waterformed sheets probably mainly by decreasing the surface tension, but dryness after the forming phase was always higher in foam forming. Higher dryness could be obtained in both cases without densification of the final dry sheet. 
The effect of foam on the structure of the fiber suspension was inherited into the final dry paper structure: formation clearly improved, which was reflected also in lower sheet roughness, and paper density decreased, which was seen in increased air permeability of the foam-formed sheets.

The z-strength of dry sheets decreased with increasing SDS dosage and with presence of foam. SDS thus seems to reduce the density and the number of interfiber contacts as well as the strength of individual contacts. The effect was smallest with the highest wet pressing pressure, probably due to the wet pressing pressure overriding the surface tension forces and the effect of bubbles.

The tensile strength and strain at break properties were determined mainly by interfiber bonding, similarly to the z-strength, being affected by both the surfactant dosage and the presence of foam.

Thus applying foam as a carrier fluid has characteristic effects both on the papermaking process and the end product properties. The main features of foam forming can be explained by the chemical effects caused by the surfactant, and the structural effects caused by the foam bubbles. However, certain effects are due to their combined effect because the foam properties and resulting sheet structure change with the surfactant dosage and applied wet pressing pressure.

Acknowledgments Open access funding provided by Technical Research Centre of Finland (VTT). The research leading to these results was conducted mainly as part of the "Efficient Networking towards Novel Products and Processes" (EffNet) research program of Forestcluster Ltd. The EffNet program received funding from Tekes-the Finnish Funding Agency for Technology and Innovation and Forestcluster Ltd. Tekes is nowadays called Business Finland. The research was also funded by VTT Technical Research Centre of Finland Ltd. Our gratitude also to Annika Ketola and Timo Lappalainen from VTT for providing the images in Figs. 2 and 23 and to Anastasia Ivanova from VTT for assisting in the graphical abstract.

Open Access This article is distributed under the terms of the Creative Commons Attribution 4.0 International License (http:// creativecommons.org/licenses/by/4.0/), which permits unrestricted use, distribution, and reproduction in any medium, provided you give appropriate credit to the original author(s) and the source, provide a link to the Creative Commons license, and indicate if changes were made.

\section{References}

Alimadadi M, Uesaka T (2016) 3D-oriented fiber networks made by foam forming. Cellulose 23(1):661-671

Aslund P, Vomhoff H (2008) Dewatering mechanisms and their influence on suction box dewatering processes: a literature review. Nord Pulp Pap Res J 23(4):389-397

Burke SR, Möbius ME, Hjelt T, Hutzler S (2019) Properties of lightweight fibrous structures made by a novel foam forming technique. Cellulose 26(4):2529-2539

Cohen-Addad S, Höhler R (2014) Rheology of foams and highly concentrated emulsions. Curr Opin Colloid Interface Sci 19(6):536-548. https://doi.org/10.1016/j.cocis.2014.11. 003

Denkov ND (2004) Mechanisms of foam destruction by oilbased antifoams. Langmuir 20(22):9463-9505

Denkov ND, Marinova KG (2006) Antifoam effects of solid particles, oil drops and oil-solid compounds in aqueous foams. In: Binks BP, Horozov TS (eds) Colloidal particles at liquid interfaces. Cambridge University Press, Cambridge, pp 383-444

Farajzadeh R, Andrianov A, Krastev R, Hirasaki GJ, Rossen WR (2012) Foam-oil interaction in porous media: implications for foam assisted enhanced oil recovery. Adv Coll Interface Sci 183-184:1-13. https://doi.org/10.1016/j.cis. 2012.07.002

Geraud B, Jones S, Cantat I, Dollet B, Meheust Y (2015) The flow of a foam in a two-dimensional porous medium. Water Resour Res 52(2):773790. https://doi.org/10.1002/ 2015WR017936

Hanson JP (1977) What's going on in non-wovens. Pulp \& Paper, pp 97-102

Haselton WR (1954) Gas adsorption by wood, pulp and paper 1. The low temperature adsorption of nitrogen, butane and carbon dioxide by sprucewood and its components. Tappi 37(9):404-412

Haselton WR (1955) Gas adsorption by wood, pulp and paper 2. The application of gas adsorption techniques to the study of the area and structure of pulps and the unbonded and bonded area of paper. Tappi 38(12):716-723

Hirasaki GJ, Lawson JB (1985) Mechanisms of foam flow in porous media: apparent viscosity in smooth capillaries. Soc Petrol Eng J 25(02):176-191. https://doi.org/10.2118/ 12129-PA

Hirt DE, Prud'homme RK, Rebenfeld L (1990) Experimental study of foam flow in fibrous materials. Tappi $\mathrm{J}$ 73(2):147-151

Hjelt T, Kinnunen K, Lehmonen J, Beletski N, Hellén E, Liljeström V, Serimaa R, Miettinen A, Kataja M (2011) Intriguing structural and strength behaviour in foam forming. In: Progress in paper physics seminar Graz

Jahangiri P, Korehei R, Zeinoddini S, Madani A, Sharma Y, Phillion A, Martinez M, Olson J (2014) On filtration and heat insulation properties of foam formed cellulose based materials. Nord Pulp Paper Res J 29(4):584-591

Jäsberg A, Selenius P, Koponen A (2015) Experimental results on the flow rheology of fiber-laden aqueous foams. Colloids Surf A 473:147-155

Jäsberg A, Selenius P, Koponen A (2017). The effect of fibrous materials on the rheology of aqueous foams. In: 
Proceedings of 16th fundamental research symposium, Robinson Pembroke College, Oxford, UK, pp 159-174

Kidner TLW (1974) The radfoam process for fine papers. Wiggins Teape Research and Development Ltd. Paper Technology, pp 346-351

Kinnunen-Raudaskoski K (2017) Foam as a carrier phase-a multipurpose technology for industrial applications. Doctoral thesis, Aalto University, Department of Bioproducts and Biosystems, Espoo, Finland

Komppa A, Komppa O (1996) Measurement of paper formation. In: 50th Appita annual general conference, Auckland, New Zealand, pp 803-808

Koponen A, Haavisto S, Salmela J, Liukkonen J (2015) Optimization of paper machine initial dewatering. Tappi $\mathbf{J}$ 14(5):325-337

Koponen A, Haavisto S, Liukkonen J, Salmela J (2016a) The flow resistance of fiber sheet during initial dewatering. Drying Technol 34(12):1521-1533. https://doi.org/10. 1080/07373937.2015.1132427

Koponen A, Torvinen K, Jäsberg A, Kiiskinen H (2016b) Foam forming of long fibers. Nordic Pulp Pap Res J 16(2):239-247

Koponen A, Ekman A, Mattila K, Al-Qararah A, Timonen J (2017) The effect of void structure on the permeability of fibrous networks. Transp Porous Media 117(2):247-259. https://doi.org/10.1007/s11242-017-0831-2

Koponen A, Jäsberg A, Lappalainen T, Kiiskinen H (2018) The effect of in-line foam generation on foam quality and sheet formation in foam forming. Nord Pulp Pap Res J 33(3):482-495. https://doi.org/10.1515/npprj-2018-3051

Kouko J, Setälä H, Tanaka A, Khakalo A, Ropponen J, Retulainen E (2018) The effect of oxyalkylation and application of polymer dispersions on the thermoformability and extensibility of paper. Carbohyd Polym 186:411-419

Kushner LM, Duncan BC, Hoffman JI (1952) A viscometric study of the micelles of sodium dodecyl sulfate in dilute solutions. J Res Natl Bur Stand 49(2):85-89

Lappalainen T, Lehmonen J (2012) Determinations of bubble size distribution of foam-fibre mixture using circular hough transform. Nord Pulp Pap Res J 27(5):930-939

Lehmonen J, Jetsu P, Kinnunen K, Hjelt T (2013) Potential of foam-laid forming technology in paper applications. Nord Pulp Pap Res J 28(3):392-398

Lehmonen J, Pere J, Hytönen E, Kangas H (2017) Effect of cellulose microfibril (CMF) addition on strength properties of middle ply of board. Cellulose 24:1041-1055

Lemlich R (1972) Adsorptive bubble separation techniques. Academic Press, New York

Lemmen JTE, Wassink JG (1990) Flow of foam through plain perforated and woven metal screens. J Soc Dyers Colour 106(7-8):235-240. https://doi.org/10.1111/j.1478-4408. 1990.tb01260.x

Li R, Du J, Zheng Y, Wen Y, Zhang X, Yang W, Lue A, Zhang L (2017) Ultra-lightweight cellulose foam material: preparation and properties. Cellulose 24(3):1417-1426

Lindqvist H, Salminen K, Kataja-aho J, Sundberg A, Holmbom B, Retulainen E (2009) Effect of electrolytes, pH and surface tension on dry and wet web properties. J Pulp Pap Sci 35(3-4): 148-154

Lobben H (1976) The tensile stiffness of paper part 2. Activation studied by freeze drying. Norsk Skogind 30(3):43-48

Lyne LM, Gallay W (1954) Studies in the fundamentals of wet web strength. Tappi J 37(12):698

Madani A, Zeinoddini S, Varahmi S, Turnbull H, Phillion AB, Olson JA, Martinez DM (2014) Ultra-lightweight paper foams: processing and properties. Cellulose 21(3):2023-2031

Page DH, Tydeman PA (1966) Physical processes occurring during the drying phase. In: Bolam F (ed) Consolidation of the paper web. Transactions of the 3rd fundamental research symposium. Cambridge, British Paper and Board Industry Research Association, London

Pöhler T, Jetsu P, Isomoisio H (2016) Benchmarking new wood fibre-based sound absorbing material made with a foamforming technique. Build Acoust 23(3-4):131-143

Punton V (1975) Fiber distribution in foam and foam-laid paper. In: International paper physics conference, pp 135-139

Radvan B, Gatward APJ (1972) The formation of wet-laid webs by a foaming process. Tappi 55(5):748-751

Rennel J (1969) Opacity in relation to strength properties of pulps. Part IV the effect of beating and wet pressing. Pulp Pap Can 70(5):151-158

Retulainen E, Ebeling K (1993) Fibre-fibre bonding and ways to characterize the bond strength. Appita 46(4):282-288

Riddell MC, Jenkins B (1976) The use of non-surfactant foaming agents in the papermachine. In: Paper technology and lndustry, pp 80-83

Saint-Jaimes A, Durian DJ (1999) Vanishing elasticity for wet foams: equivalence with emulsions and role of polydispersity. J Rheol 43(6):1411-1422. https://doi.org/10.1122/ 1.551052

Skelton J (1987) Foam assisted dewatering — a new technology emerges. Paper Technology and Industry, pp 431-436

Smith MK, Punton VW (1975) Foam can improve formation. Pulp Paper Canada 76(1):55-58

Smith MK, Punton VW, Rixson AG (1974) The structure and properties of paper formed by a foaming process. Tappi 57(1):107-111

Thondavadl N, Lemllich R (1985) Flow properties of foam with and without solid particles. Ind Eng Chem Process Des Dev 24(3):748-753

Torvinen K, Lahtinen P, Kiiskinen H, Hellen E, Koponen A (2015). Bulky paper and board at a high dry solids content with foam forming. In: Proceedings of Papercon 2015, Atlanta, Georgia, pp 757-766

Wiggins T (1973) Make paper with foam not without it. A report from Wiggins Teape Research and Development Ltd. Pulp Trade J 16:28-31

Publisher's Note Springer Nature remains neutral with regard to jurisdictional claims in published maps and institutional affiliations. 